
\title{
Research Suare \\ The neuroprotective effects of AMN082 on neuronal apoptosis in rat after traumatic brain injury
}

Chung-Che Lu

Chi Mei Medical Center

Che-Chuan Wang

Chi Mei Medical Center

Yao-Lin Lee

Chi Mei Medical Center

Chung-Ching Chio

Chi Mei Medical Center

Sher-Wei Lim

Chi Mei Medical Center

Jinn-Rung Kuo ( $\square$ kuojinnrung@gmail.com )

Chi Mei Medical Center

\section{Research Article}

Keywords: AMN082, Traumatic brain injury, Nitrosative stress, Apoptosis, NMDA receptor

Posted Date: December 15th, 2020

DOI: https://doi.org/10.21203/rs.3.rs-121579/v1

License: (c) (i) This work is licensed under a Creative Commons Attribution 4.0 International License.

Read Full License 


\section{Abstract}

The aim of this study is to investigate whether the neuroprotective effect of AMN082 is via attenuating glutamic receptor associated neuronal apoptosis and improves functional outcomes after traumatic brain injury (TBI). Anesthetized male Sprague-Dawley rats were divided into sham-operated, $\mathrm{TBI}+$ vehicle, and TBI + AMN082 groups. AMN082 was intraperitoneally injected $(10 \mathrm{mg} / \mathrm{kg})$ at 0, 24, and $48 \mathrm{hr}$ after TBI. During the 120 minutes after TBI, heart rate, mean arterial pressure, intracranial pressure (ICP), and cerebral perfusion pressure (CPP) were continuously measured. The motor function, infarction volume, and neuronal nitrosative stress-associated apoptosis, N-Methyl-D-aspartate receptor 2A (NR2A) and NR2B expression were measured on the 3rd day after TBI. The results showed AMN082-treated group had the lower ICP and higher CPP after TBI. The TBI-induced motor deficits, increased infarction volume, neuronal apoptosis, 3-nitrotyrosine and inducible nitric oxide synthase expression in the peri-contusion cortex were significantly improved by AMN082 therapy. Simultaneously, AMN082 increased the NR2A and NR2B expression in neuronal cells. We concluded intraperitoneal injection of AMN082 for 3 days may ameliorate TBI insults by attenuating glutamic receptor associated nitrosative stress and neuronal apoptosis in the peri-contusion cortex. We suggest AMN082 administration in acute stage may be a promising strategy for TBI.

\section{Introduction}

Excessive glutamate excitotoxicity is a well-known leading cause of neurological complications after traumatic brain injury (TBI). ${ }^{1}$ Metabotropic receptors (mGlus) and ionotropic receptors, which include Nmethyl-D-aspartate receptor 2A (NR2A) and 2B (NR2B), are two major glutamic receptors in the central nervous system (CNS). 2, 3 Triggering astromicroglial and neuronal glutamate excitotoxicity generates inducible nitric oxide synthase (iNOS) is expressed and reactive nitrosative stress (RNS) occurred, and eventually contributing to cell apoptosis. ${ }^{4,5}$ Therefore, inhibition of glutamate-related RNS processes may play a role as a therapeutic strategy after TBI.

AMN082 ( $\mathrm{N}, \mathrm{N}$-dibenzhydrylethane-1, 2-diamine dihydrochloride) is a blood-brain barrier permeable and a highly selective $\mathrm{mGLu} 7$ receptor allosteric agonist with clear pharmacokinetic activities. ${ }^{6}$ Its metabolite Met- 1 has approximately 100 -fold greater affinity than AMN082 for binding to the mGlu 7 receptor. ${ }^{7}$ Its action on the CNS has demonstrated anti-anxiety and antidepressive effects ${ }^{8-10}$ and antiparkinsonianlike effects, ${ }^{11}$ and it is used in the treatment of cocaine or opioid addiction. ${ }^{12}$ These mechanisms include regulating glutamate release at the synapse, ${ }^{13}$ binding to the serotonin transporter, ${ }^{6}$ activation of prosurvival MAPK/ERK $1 / 2$ and PI3-K/Akt pathways, ${ }^{14}$ and stimulated mammalian target of rapamycin (mTOR) activation. ${ }^{9}$ However, to date AMN082 has not been applied in TBI. The effects of AMN082 on neurons and glia as well as TBI-induced nitrosative stress-associated neuronal apoptosis have not been well investigated. 
In the current study, we hypothesized that AMN082 would have therapeutic effects on TBI because it is a selective mGlu7 receptor that affects glutamate release. We aimed to elucidate the beneficial effects of AMN082 on TBI-induced nitrosative stress-related apoptosis. We hope our results may provide new information that AMN082 may play a role in clinical application in the field of neurotrauma in the future.

\section{Results}

\section{Basic data of experimental rats}

A total of 48 male rats, 10 - week-old weighing $413 \pm 8.7 \mathrm{~g}$ were used in the experiments. The fluid percussion force was $2.20 \pm 0.01 \mathrm{~atm}$. The colonic temperature was controlled around $36-37^{\circ} \mathrm{C}$ with lamp insulation during and up to 120 min after injury. No animals died over the course of the experiments.

\section{AMN082-treated group had the trend of lower ICP and higher CPP during the initial 120minutes after TBI}

In the TBI group, the ICPs were higher at 0-120 minutes after the start of FPI than they were for shamoperated controls. In contrast, the values for CPP were significantly lower than those of sham-operated controls, ${ }^{*} p<0.05, n=6$ in sham and $n=6$ in the TBI group (Fig. $2 a-2 d$ ).

\section{Treatment with AMN082 improved TBI-induced motor impairment at the $3^{\text {rd }}$ day after TBI}

The maximal grip angle on the $3^{\text {rd }}$ day after TBI was significantly lower compared with sham controls $\left(45.3^{\circ} \pm 0.72^{\circ}\right.$ versus $53.9 \pm 0.37^{\circ}$, respectively, $\left.{ }^{\star *} \mathrm{p}<0.001\right)$. The TBI-induced motor impairment was significantly improved by AMN082 treatment (TBI group versus AMN082 group, $45.3^{\circ} \pm 0.72^{\circ}$ versus $47.5 \pm 0.34, \$ p<0.05$; Figure 3$), n=6$ in each group.

Treatment with AMN082 significantly improved the TBI-induced cerebral infarction volume on the $3^{\text {rd }}$ day after TBI

Compared with the sham group, the TBI group showed significantly increased infarct volume (134.4 \pm $3.93 \mathrm{~mm} 3$ versus $\left.0 \pm 0 \mathrm{~mm}^{3}, * \star * \mathrm{P}<0.001\right)$. However, the TBI-treated with AMN082 group exhibited significantly reduced infarct volume compared with the TBI group $(66.9 \pm 19.62 \mathrm{~mm} 3$ versus $134.4 \pm 3.93$ $m m 3, \# P<0.05$, Figure 4), $n=6$ in each group.

\section{Treatment with AMN082 significantly attenuated neuronal apoptosis in the peri-contusion cortex at $3^{\text {rd }}$ day after $\mathrm{TBI}$}

Using the Caspase-3 and TUNEL assay on the 3rd day after TBI, we found that the number of apoptotic neuronal cells (Neu-N plus Caspase- 3 and Neu-N plus TUNEL staining assays) in the peri-contusion cortex was significantly increased compared with that of the sham rats ( $* \star * P<0.001)$. The number of positive apoptotic cells in rats with TBI was significantly reduced after AMN082 treatment $\left(\# P<0.05,{ }^{\# \#} P<0.01\right.$, Figure 5, 6), n=6 in each group. 
Treatment with AMN082 significantly improved neuronal nitrosative stress in the peri-contusion cortex on the $3^{\text {rd }}$ day after TBI

Compared with the sham controls $(62.1 \pm 19.87)$, the number of positive n-NOS plus Neu-N-stained (37.6 \pm 4.99 , Table 2) cells in the peri-concussion cortex of TBI rats was non-significantly decreased. In the 3NT plus Neu-N-stained assay, the number of positive neuronal 3-NT cells in the peri-contusion cortex of vehicle-treated rats was significantly increased compared with the sham controls ( $56.0 \pm 4.35$ versus $0 \pm 0$ ). The TBI-induced decrease in the number of neuronal n-NOS and increase in the number of 3-NT positive cells was significantly improved by AMN082 therapy (Table 2).

Treatment with AMN082 significantly improved glialnitrosative stress in the peri-contusion cortex on the

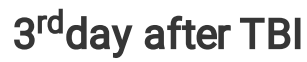

Compared with the TBI rats, the TBI-induced iNOS in the activated microglia $(29.6 \pm 3.92, * \star * p<0.001$, Table 2$)$ and astrocytes $(28.2 \pm 3.48, \star \star \star p<0.001$, Table 2$)$ was significantly lower in the AMN082-treated rats $(17.9 \pm 3.14 ; 19.1 \pm 2.06$, $\# p<0.05$, respectively), $n=6$ in each group (Table 2$)$.

\section{AMN082-treated groups had a higher ratio of NR2AVNR2B than the TBI rats in the peri-contusion cortex}

Compared with those in the sham controls $(0 \pm 0)$, the number of positive NR2A plus Neu-N-stained cells $\left(27.1 \pm 2.74,{ }^{* \star *} \mathrm{p}<0.001\right)$ in the peri-contusion cortex of TBI rats was significantly increased. AMN082treated groups exhibited further significantly increased numbers of neuronal NR2A positive cells after TBI $\left(50.0 \pm 4.18,{ }^{\# \#} \mathrm{P}<0.01\right.$, Table 2$), \mathrm{n}=6$ in each group.

Compared with the TBI rats, the number of TBI-induced NR2B plus Neu-N-stained cells $(33.6 \pm 4.76, n=6$, Table 2) was significantly lower than in the sham $(101.1 \pm 4.03, \star \star \star p<0.001, n=6)$ and AMN082-treated rats $(45.7 \pm 2.47, \# p<0.05, n=6)$.

We further tested the NR2A/NR2B ratio in TBI vehicle-treated rats and TBI AMN082-treated groups, and we found that the TBI AMN082-treated groups had a higher ratio than the TBI vehicle-treated rats (1.09 \pm 0.08 versus $0.89 \pm 0.16$ ) (Table 2 ).

\section{Discussion}

\section{Summary of the current study}

According to our review of the literature, this study is the first to demonstrate the effects of AMN802 on TBI. In this study, we added new information to the field of traumatology. We found that treatment with AMN802 could improve neuropathology and functional outcome after TBI. At the cellular level, ANMN082 experienced less iNOS and 3-NT expression, less neuronal apoptosis, and higher NR2A/2B glutamic receptor expression in astromicroglia or neuronal cells. In functional outcomes, the TBI-treated group had very significantly increased motor function. Therefore, we suggest that AMN082 may be a promising 
treatment strategy in critical care for the TBI-induced nitrosative stress associated neuronal apoptotic pathway that leads to functional outcome impairment.

\section{Dosage and time course of AMN082 treatment}

There were several dosages and temporal administration methods of AMN082 in neurobehavior field. AMN082 (1 mg/kg, I.P.) exerted significant antidepressant-like effects in the tail suspension test. ${ }^{15}$ AMN082 (1 and $3 \mathrm{mg} / \mathrm{kg}, \mathrm{I}$. .P.) decreased the haloperidol $(0.25 \mathrm{mg} / \mathrm{kg})$-induced parkinsonian-like effects. ${ }^{11}$ AMN082 at doses of 2.5 and $5 \mathrm{mg} / \mathrm{kgl} . P$. also decreased ethanol- and morphine withdrawal-induced anxiety-like behavior in the elevated plus maze test. ${ }^{10}$ AMN082 at a dose of $10 \mathrm{mg} / \mathrm{kg}$ I.P. suppressed the locomotor effect of both cocaine- or morphine-induced hyperactivity. ${ }^{12}$ When AMN082 was injected I.P. at a constant volume of $10 \mathrm{ml} / \mathrm{kg}$, activation of the mGlu7 receptor elicited antidepressant-like effects. ${ }^{16}$ In the current study, we gave $10 \mathrm{mg} / \mathrm{kg}$ of AMN082 for three consecutive days after TBI. These dosages and the observation end-point 3 days after TBI were selected because lateral fluid percussion causes motor dysfunction from 3 days to 1 year after $\mathrm{TB},{ }^{17}$ and considerable evidence suggests that brain edema after $\mathrm{TBI}$ is maximal at $2-3$ days after $\mathrm{TBI}$, which is usually also the peak point of ICP. ${ }^{18}$ Furthermore, we did not find any side effect during treatment course. Our positive results support the idea that AMN082 at this dose may have therapeutic benefits in TBI episodes.

\section{Early effects of AMN082 on physiological parameters during the initial 120minutes after TBI}

In the current study, compared to the TBI groups, it indicated that the AMN082-treated group $(10 \mathrm{mg} / \mathrm{kg}$, i.p.) had the trend of lower ICP and higher CPP mostly happened around 0 min during the initial 120minutes after TBI. We consider that these early beneficial effects of AMN082 on ICP and CPP may lead to attenuated secondary injury process development and improvement outcome on the $3^{\text {rd }}$ day after TBI. Sukoff Rizzo et al have demonstrated that following a single injection of $10 \mathrm{mg} / \mathrm{kg}$ (i.p.), AMN082 was found to cross the blood brain barrier with peak brain concentrations at 30 min post treatment. ${ }^{6}$ However, we did not investigate the relationship between the brain AMN082 concentration and physiological parameters changes after TBI. This relationship warrants further investigation.

\section{Possible mechanisms of action of AMN082on TBI-induced neuropathological changes and functional outcome}

Inducible NOS produced large amounts of nitric oxide (NO) followed by 3-NT formation, leading to cell apoptosis. ${ }^{5,19}$ Our results found that the expression of iNOS was increased in microglia and astrocytes in the TBI group and was attenuated in AMN082-treated TBI groups. This nitrosative stress associated neuronal apoptosis was consistent with peroxynitrite inhibited caspase-mediated effects for in vivo and in vitro TBI models; ${ }^{19}$ treatment with brain cooling ${ }^{5}$ or memantine ${ }^{4}$ could attenuate brain nitrosative damage, lower the infarction volume of the cortex and improve functional outcomes after TBI, and coculture with 3 NT induced motor neuron apoptosis. ${ }^{20}$ 
The glutamic receptor NR2A is involved in neuroprotection, and NR2B triggers cellular destructive pathways. Both NR2A and NR2B are mostly located in neurons. ${ }^{2,3}$ To date, the expression of NR2A and NR2B in AMN082 therapy was not investigated in the TBI field. In the current study, we found that both NR2A and NR2B were significantly increased in AMN082-treated groups after TBI, and TBI-AMN082treated groups had a higher ratio than the TBI vehicle-treated rats. Therefore, we consider that AMN082 may affect NR2A and NR2B expression, although AMN082 is a highly selective mGlu7 receptor agonist. However, the detailed mechanisms need to be investigated in the future.

Neuronal nitric oxide synthase (nNOS) constitutes the predominant source of NO in neurons and has dual biological activities. ${ }^{21}$ Its activity is related to NMDA receptor mediated excitotoxicity. ${ }^{22}$ In the current study, we found thatAMN082 increased the nNOS expression, which was parallel to both neuronal 3NT and apoptosis reduction, and higher ratios of NR2A/ER2B. In such conditions, nNOS may play an important role for neuroprotection.

Therefore, we infer that there were several possible mechanisms of action that may be involved. 1. AMN082, a highly selective mGlu7 receptor agonist, exerts an inhibitory effect on presynaptic glutamate release from rat cerebro-cortical nerve terminals. ${ }^{4,7}$ Therefore, its reduction of glutamate release to postsynaptic space or extrasynaptic space appears to be an efficient way to inhibit the nitrosative stress development and achieve neuroprotection. 2. AMN082 with postsynaptic mGlu7 receptor could modulate NMDA receptor activity ${ }^{8,23}$ or directly bind to NMDA receptors such as NR2A and NR2B receptors, resulting in knows neuroprotection. ${ }^{4,6} 3$. AMN082 binds to the mGlu7 receptor in astrocytes and microglia, ${ }^{14}$ and attenuates TBI-induced iNOS expression in activated microglia and astroglia in the pericontusion cortex. These possible mechanisms may result in neuronal 3NT apoptosis reduction and finally improve functional outcomes for TBI. Although there are various possibilities, the results show that AMN082 is effective and relevant for TBI treatment, particularly in leading to neuroprotection via attenuation of brain nitrosative stress-associated apoptosis, which is a serious secondary injury after TBI.

\section{Limitations of the current study}

Several drawbacks of the current study should be mentioned. First, we only investigated the subgroup of NMDA receptor named NR2A and NR2B without detecting kainite or a-amino-3-hydroxy-5 methyl-4isoxazole propionic acid (AMPA) that may influence nitrosative stress and apoptosis after TBI. Second, we just proof the associations between the parameters at the specific time point 3 days after TBI. Third, we only detect the effects of AMN082 on male rats; it should be clarified whether these results can translate to female rat or other species or application to clinical practice or not in the future. Fourth, we only use a single functional method, an inclined plane test which was based on this test can measure motor functions after neural damage in rats. However, our results showed a small absolute difference in angle between TBI + vehicle and TBI + AMN082 groups. Whether these results actually suggest that there might be a clinically meaningful difference in humans needs further confirmation. Also, it would be used multiple tests for the motor function in the future. Finally, this study, there were 3 groups: sham-operated group, TBI + vehicle group and TBI + AMN082 group. Based on the AMN082 has not been applied in TBI, 
the novel information of AMN082 effects on neurons and glia as well as TBI-induced nitrosative stressassociated neuronal apoptosis and neuronal NR2A/2B expression may provide to critical care physician in the clinical practice. However, we could consider adding another group: TBI + AMN082 antagonist group such as 6-(4-methoxyphenyl)-5-methyl-3-pyridinyl-4-isoxazolo [4, 5-c] pyridin-4(5H)- one (MMPIP), ${ }^{24}$ a selective mGluR7 antagonist, which will make the study more impeccable and convincible. Therefore, many more studies are required in the future.

\section{Conclusion}

Our results demonstrated that intraperitoneal administration of AMN082 for three days after TBI may ameliorate TBI insults by affecting glutamic receptors related nitrosative stress, NR2A/2B expression, and decreasing neuronal apoptosis in the injured cortex. These mechanisms might represent why functional recovery may occur. We also consider that AMN082 may be a promising treatment strategy in acute stage in critical care after TBI.

\section{Material And Methods}

\section{Experimental design}

The overall experimental protocol is shown in Figure 1. First, we continuously measured the physiological changes, including heart rate (HR), mean arterial pressure (MAP), intracranial pressure (ICP) and cerebral perfusion pressure (CPP) for 120 minutes after TBI. Then, we tested whether TBI effects were associated with nitrosative stress related neuronal apoptosis, cerebral infarction in the ipsilateral injury side of cortex using immunofluorescence and TTC staining. The motor function was examined by incline plane on the $3^{\text {rd }}$ day after TBI. During the same time course, the effects of AMN082 on TBI association parameters were also evaluated. All methods were carried out in accordance with relevant guidelines and regulations.

\section{Animals}

Ten-week-old adult male Sprague-Dawley rats were used in the experiments. The animals were kept under a 12/12-h light/dark cycle and allowed free access to food and water. The Chi Mei Medical Center's Animal Care and Use Committee approved all of the experimental procedures, which conformed to the $\mathrm{NIH}$ guidelines, including minimizing discomfort to the animals during surgery and during the recovery period. At the end of the experiments, rats were sacrificed with an overdose of urethane.

The study was carried out in compliance with the ARRIVE guidelines.

\section{Traumatic brain injury}

Animals were anesthetized with a mixture of ketamine $(44 \mathrm{mg} / \mathrm{kg}$, i.m.; Nankuang Pharmaceutical, Taiwan), atropine (0.063 mg/kg, i.m.; Sintong Chemical Ind. Co., Taiwan), and xylazine (6.77 mg $/ \mathrm{kg}$, i.m.; Bayer, Germany). On a stereotaxic frame (Kopf 1406; Grass Instrument, Quincy, MA), craniectomy was 
performed with $2 \mathrm{~mm}$ in radius, $3 \mathrm{~mm}$ from the sagittal sutures and $4 \mathrm{~mm}$ from the bregma in the right parietal cortex. After implantation of the injury cannula, the fluid percussion device (VCU Biomedical Engineering, Richmond, VA, USA) was connected to the craniectomy site via Luer-lock fitting, and the brain was injured with 2.2atm, 25 ms percussion as originally described by McIntosh et al. ${ }^{25}$ Selection criteria of successful TBI insult of rat were transient hypertensive response, apnea, and seizure observed immediately following fluid percussion injury.

\section{Surgery and physiological parameters monitoring}

The right femoral artery of rats was cannulated with polyethylene tubing (PE50) under for blood pressure monitoring. Mean arterial pressure (MAP) and heart rate (HR) were monitored continuously with a pressure transducer after TBI. An intracranial pressure (ICP) microsensor (Codman and Shurtlef, Inc., Rayman, MA, USA) was placed in the parenchyma of the left frontal lobe of each rat. ICP was monitored continuously, and ICP and cerebral perfusion pressure (CPP) values were recorded at 5-minute intervals in the period of 120 minutes after TBI. The CPP value is defined as MAP-ICP. ${ }^{26}$ Colon temperatures were measured with an analog electronic thermometer (model 43 TE; YSI, Inc, Yellow Springs, $\mathrm{OH}$ ) and temperature probe (series 400; YSI, Inc).

\section{Treatment intervention}

Using the random number table, the rats were numbered in random order and assigned to three major groups: sham operated $(n=6)$; TBI control treated with vehicle, dimethylsulfoxide (DMSO) $(4 \%, 1 \mathrm{ml} / \mathrm{kg}$, injected intraperitoneally, K42088831, vehicle; Merck, Darmstadt, Germany); and TBI +AMN082-treated (10 mg/kg, dissolved with DMSO, injected intraperitoneally; U. S. Pharmacopeia) $(n=6)$. The TBI without treatment group received equal volumes of vehicle via intraperitoneal injection. Vehicle or AMN082 was administered for three consecutive days after TBI. The first injections occurred immediately after TBI, the second injection was administered $24 \mathrm{~h}$ later, and the third injection was administered $48 \mathrm{~h}$ later. All tests were performed with operators and assessors blinded to the study rats, which were revealed only at the end for the analyses.

\section{Motor function test}

An inclined plane was used to measure limb strength for motor function test. ${ }^{27}$

This test can measure the motor function of rats after neural injury by evaluating the rat's ability to prevent falls and the endurance strength of the upper and lower limbs in an inclined plane. The animals were initially placed on a $30^{\circ}$ angle, $20 \times 20 \mathrm{~cm}$ buffer ribbed surface incline plane. To determine the maximal angle at which an animal could remain on the incline plane without drop, the angle was increased in $1^{\circ}$ increments. Motor deficit were measured with the mean maximal angles of the left upper and lower limbs on the $3^{\text {rd }}$ day after TBI.

\section{Cerebral infarction assay}


The triphenyltetrazolium chloride (TTC) staining procedures followed those described elsewhere. ${ }^{28}$ Briefly, the brain tissue was removed, immersed in cold saline for $5 \mathrm{~min}$, and sliced into $1 \mathrm{~mm}$ sections. The brain slices were incubated in $2 \%$ TTC dissolved in phosphate-buffered saline for $30 \mathrm{~min}$ at $37^{\circ} \mathrm{C}$ and then transferred to $10 \%$ formaldehyde solution for fixation. The volume of infarction, as revealed by negative TTC stains indicating dehydrogenase-deficient tissue, was measured in each slice and summed using computerized planimetry (PC-based Image Tools software). The volume of infarction was calculated as 1 $\mathrm{mm}$ (thickness of the slice) $\mathrm{x}$ (sum of the infarction area in all brain slices $\left[\mathrm{mm}^{2}\right]$ ). ${ }^{29}$

\section{Immunofluorescence assay}

Immunofluorescence assay was measured on the $3^{\text {rd }}$ day after TBI. ${ }^{30}$ Adjacent $6-\mu \mathrm{m}$ sections corresponding to coronal coordinates $0.20 \mathrm{~mm}$ to $0.70 \mathrm{~mm}$ anterior to bregma were incubated in $2 \mathrm{~mol} / \mathrm{L}$ $\mathrm{HCl}$ for $30 \mathrm{~min}$, rinsed in $0.1 \mathrm{~mol} / \mathrm{L}$ boric acid $(\mathrm{pH} 8.5)$ for $3 \mathrm{~min}$ at room temperature, and then incubated with primary antibodies in PBS containing $0.5 \%$ normal bovine serum at $4^{\circ} \mathrm{C}$ overnight. After being washed in PBS, the sections were incubated with secondary antibodies for $1 \mathrm{~h}$ at room temperature. The number of immunofluorescent positive cells was calculated using computerized planimetry corresponding to the peri-contusion cortical region (400 x magnification, Image-Pro Plus Media Cybernetics, Inc. Washington Street, Rockville, USA), and the number of positive cells was expressed per $\mathrm{mm}^{2}$ in interested region. The detailed antibodies used were summarized on Table 1.

\section{Statistical analysis}

According to the ANOVA with the level of type I error at 0.05 and the smallest effect size at 0.2 for power calculation, so the sample size available will be at least 6 rats in each groups. This proposed sample size is sufficient to detect a statistical significant of ANOVA at the 0.05 level with power of $80 \%$. All of the data were analyzed using SigmaPlot, version 10.0 for Windows (Systat Software, San Jose, CA) in this study. The results are expressed as the means \pm standard deviation of the means for the experiments. For each time points, the mean difference between these three groups was estimated using the analysis of variance (ANOVA). The analysis of variance (ANOVA) with post hoc Scheffe's test was used to estimate the statistical difference between these three groups. P-values $<0.05$ were considered statistically significant.

\section{Declarations}

\section{Author Contributions}

S-W $L$ and J-R K conceived and designed the experiments. C-C $L$ and $Y-L L$ performed the experiments. SW L and J-R K analyzed the data. C-C C and C-C W contributed reagents/materials/analysis tools. J-R K and S-W L wrote the paper. All authors reviewed the manuscript.

\section{Acknowledgments}


The authors thank Chiao-Ya Hu, who participated in this study.

\section{References}

Mantovani, A., Allavena, P., Sica, A. \& Balkwill, F. Cancer related inflammation. Nature. 454, 436-444 (2008).

1. Lipton, S.A., Rosenberg PA. Excitatory amino acids as a final common pathway for neurologic disorders. N Engl J Med. 330: 613-622 (1994).

2. Dingledine, R., Borges, K., Bowie, D., Traynelis, S.F. The glutamate receptorion channels. Pharmacol Rev. 51: 7-61 (1999).

3. Brassai, A., Suvanjeiev, R.G., Bán, E.G., Lakatos, M. Role of synaptic and nonsynaptic glutamate receptors in ischaemia induced neurotoxicity. Brain Res Bull. 112: 1-6 (2015).

4. Wang, C.C., Wee, H.Y., Hu, C.Y., Chio, C.C., Kuo, J.R. The Effects of Memantine on Glutamic ReceptorAssociated Nitrosative Stress in a Traumatic Brain Injury Rat Model. World Neurosurg. 112:e719e731 (2018).

5. Kuo, J.R., Lo, C.J., Chang, C.P., Lin, M.T., Chio, C.C. Attenuation of brain nitrostative and oxidative damage by brain cooling during experimental traumatic brain injury. J Biomed Biotechnol. 2011: 145214 (2011).

6. Sukoff Rizzo et al. The metabotropic glutamate receptor 7 allosteric modulator AMN082: a monoaminergic agent in disguise? J Pharmacol Exp Ther. 2011; 338: 345-352 (2011).

7. Mitsukawa, K. et al. A selective metabotropic glutamate receptor 7 agonist: activation of receptor signaling via an allosteric site modulates stress parameters in vivo. Proc Nat Acad Sci USA. 102:18712-18717 (2005).

8. Bradley, S.R., Uslaner, J.M., Flick, R.B., Lee, A., Groover, K.M., Hutson, P.H. The mGluR7 allosteric agonist AMN082 produces antidepressant-like effects by modulating glutamatergic signaling. Pharmacol Biochem Behav. 101:35-40 (2012).

9. Pałucha-Poniewiera, A., Szewczyk, B., Pilc, A. Activation of the mTOR signaling pathway in the antidepressant-like activity of the mGlu5 antagonist MTEP and the mGlu7 agonist AMN082 in the FST in rats. Neuropharmacology. 82: 59-68 (2014).

10. Kotlinska, J.H. et al. Impact of the metabotropic glutamate receptor7 (mGlu7) allosteric agonist, AMN082, on fear learning and memory and anxiety-like behavior. Eur J Pharmacol. 858:172512 (2019).

11. Konieczny, J, Lenda, T. Contribution of the mGluR7 receptor to antiparkinsonian-like effects in rats: a behavioral study with the selective agonist AMN082. Pharmacol Rep. 65:1194-1203 (2013).

12. Jenda, M., Gawel, K., Marszalek, M., Komsta, L., Kotlinska, J.H. AMN082, a metabotropic glutamate receptor 7 allosteric agonist, attenuates locomotor sensitization and cross-sensitization induced by cocaine and morphine in mice. Prog Neuropsychopharmacol Biol Psychiatry. 57:166-175 (2015). 
13. Wang, C.C., Kuo, J..R, Huang, S.K., Wang, S.J. Metabotropic glutamate 7 receptor agonist AMN082 inhibits glutamate release in rat cerebral cortex nerve terminal. Eur J Pharmacol. 823:11-18 (2019).

14. Jantas, D., Greda, A., Golda, S., Korostyriski, M., Lason, W. The neuroprotective effects of prthostericagnonists of group II and III mGLuRs in primary neuronal ccells cultures are dependent on developmental stage. Neuropharmacology. 111:195-211 (2016).

15. Podkowa, K., Pilc, A., Podkowa, A., Sałat, K., Marciniak, M., Pałucha-Poniewiera, A. The potential antidepressant action and adverse effects profile of scopolamine co-administered with the mGlu7 receptor allosteric agonist AMN082 in mice. Neuropharmacology. 141:214-222 (2018).

16. Palucha, A., Klak, K., Branski, P., van der Putten, H., Flor, P.J., Pilc, A. Activation of the mGlu7 receptor elicits antidepressant-like effects in mice. Psychopharmacology (Berl). 194:555-562 (2007).

17. Pierce, J.E., Smith, D.H., Trojanowski, J.Q., McIntosh, T.K. Enduring cognitive, neurobehavioral and histopathological changes persist for up to one year following severe experimental brain injury in rats. Neuroscience. 87:359-369 (1998).

18. Marmarou, A. Pathophysiology of traumatic brain edema: current concepts. Acta Neurochir Suppl. 86:7-10 (2003).

19. Lau, A., Arundine, M., Sun, H.S., Jones, M., Tymianski, M. Inhibition of Caspase-Mediated Apoptosis by Peroxynitrite in Traumatic Brain Injury. J Neurosci. 26:11540-11553 (2006).

20. Peluffo, H. et al. Induction of motor neuron apoptosis by free 3-nitro-L-tyrosine. J Neurochem. 89:602-612 (2004).

21. Zhou, L., Zhu, D.Y. Neuronal nitric oxide synthase: structure, subcellular localization, regulation, and clinical implications. Nitric Oxide. 20: 223-230 (2009).

22. Eliasson, M.J. et al. Neuronal nitric oxide synthase activation and peroxynitrite formation in ischemic stroke linked to neural damage, J. Neurosci. 19:5910-5918 (1999).

23. Gu, Z., Liu, W., We, J., Yan, Z. Regulation of N-methyl-D-aspartic acid (NMDA) receptors by metabotropic glutamate receptor 7. J Biol Chem. 287, 10265-10275 (2012).

24. Palazzo, E. et al. MMPIP, an mGluR7-selective negative allosteric modulator, alleviates pain and normalizes affective and cognitive behavior in neuropathic mice. Pain. 156:1060-1073 (2015).

25. Mclntosh, T.K. et al. Traumatic brain injury in the rat: characterization of a lateral fluid-percussion model. Neuroscience. 28:233 (1998).

26. The Brain Trauma Foundation. The American Association of Neurological Surgeons. The Joint Section on Neurotrauma and Critical Care. Cerebral perfusion pressure thresholds. J Neurotrauma. 24: S59-64 (2007).

27. Hallam, T.M. et al. Comparison ofbehavioral deficits and acute neuronal degeneration in ratlateral fluid percussion and weight-drop brain injury models. J Neurotrauma. 21:521539 (2004).

28. Kuo, J.R., Lo, C.J., Chio, C.C., Chang, C.P., Lin,. MT. Resuscitation from experimental traumatic brain injury by agmatine therapy. Resuscitation. 75: ,506-514 (2007) 
29. Wang, Y, Lin, S..Z, Chiou, A.L., Williams, L.R., Hoffer, B.J. Glial cell line-derived neurotrophic factor protects against ischemia induced injury in the cerebral cortex. J Neurosci. 17:4341-4348 (1997).

30. Chong, A.J., Lim, S.W., Lee, Y.L., Chio, C.C., Chang, C.H., K.uo, J.R., Wang, C.C. World Neurosurg. The neuroprotective effects of simvastatin on high cholesterol following traumatic brain injury in rats. 2019;132:e99-e108.

\section{Tables}

Table 1 The detailed information of antibodies used in current study

\begin{tabular}{cccc}
\hline \multicolumn{1}{l}{ Antibody } & Source & Catalog Number & Working Dilution \\
\hline Mouse anti-NeuN & Abcam(Cambridge, MA) & Ab104224 & $1: 600$ \\
Mouse anti-OX42 & Abcam(Cambridge, MA) & ab1211 & $1: 200$ \\
Mouse anti-GFAP & Cell signaling technology(Beverly, MA) & 3670 & $1: 800$ \\
Rabbit anti-Caspase3 & Cell signaling technology(Beverly, MA) & 9661 & $1: 500$ \\
TUNEL kit & Clontech(Palo Alto, CA) & 630108 & \\
Rabbit anti-nNOS & Invitrogen(Eugene,Oregon) & PA3-032A & $1: 200$ \\
Rabbit anti-iNOS & Abcam(Cambridge, MA) & Ab15323 & $1: 200$ \\
Mouse anti-3NT & Abcam(Cambridge, MA) & ab61392 & $1: 200$ \\
Rabbit anti-NMDAR2A & Chemicon international(Billerica, MA) & AB1555 & $1: 600$ \\
Rabbit anti-NMDAR2B & Abcam(Cambridge, MA) & ab65783 & $1: 500$ \\
\hline
\end{tabular}


Table 2 AMN082 significantly attenuate nitrosative stress, neuronal apoptosis and NMDA2A/2B expression at 72 hrs following TBI

\begin{tabular}{|c|c|c|c|}
\hline Parameters / Groups & Sham & TBI+vehicle & TBI+AMN082 \\
\hline Number of NeuN-nNOS positive cells $\left(\mathrm{mm}^{2}\right)$ & $62.1 \pm 19.87$ & $37.6 \pm 4.99$ & $57.9 \pm 5.74^{\#}$ \\
\hline Number of OX42-iNOS positive cell $\mathrm{s}\left(\mathrm{mm}^{2}\right)$ & $0 \pm 0$ & $29.6 \pm 3.92^{* k * ⿰ ㇇ ⿰ 亅 ⿱ 丿 丶 丶 ~}$ & $17.9 \pm 3.14^{* k * k, ~} \#$ \\
\hline Number of GFAP-iNOS positive cells $\left(\mathrm{mm}^{2}\right)$ & $0 \pm 0$ & $28.2 \pm 3.48^{\text {水水 }}$ & $19.1 \pm 2.06^{*} k_{k}, \#$ \\
\hline Number of NeuN-3NT positive cells $\left(\mathrm{mm}^{2}\right)$ & $0 \pm 0$ & $56.0 \pm 4.35^{\text {水水 }}$ & 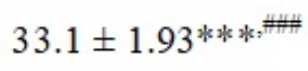 \\
\hline Number of NeuN-NMDAR2A positive cells $\left(\mathrm{mm}^{2}\right)$ & $0 \pm 0$ & $27.1 \pm 2.74^{\text {k } k_{k} *}$ & 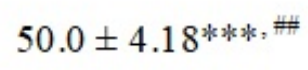 \\
\hline Number of NeuN-NMDAR2B positive cells $\left(\mathrm{mm}^{2}\right)$ & $101.1 \pm 4.03$ & $33.6 \pm 4.76^{\text {*k } k_{k} *}$ & $45.7 \pm 2.47$ 水水, \\
\hline NMDAR2A / NMDAR2B & $0 \pm 0$ & $0.89 \pm 0.16^{\text {*k } k_{k} *}$ & $1.09 \pm 0.08^{*}$ *k $k_{k}$ \\
\hline
\end{tabular}

*** $\mathrm{P}<0.001$, compared with the Sham group

\# $\mathrm{P}<0.05$, \#\# $\mathrm{P}<0.01$, \#\#\# $\mathrm{P}<0.001$, compared with the TBI+vehicle group

Figures 
(A)

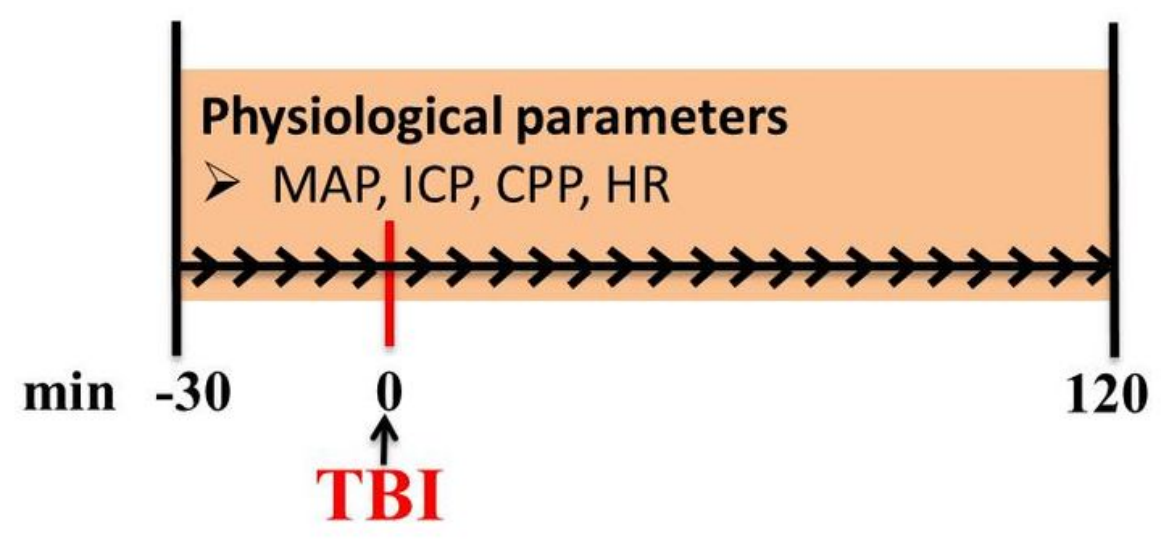

(B)

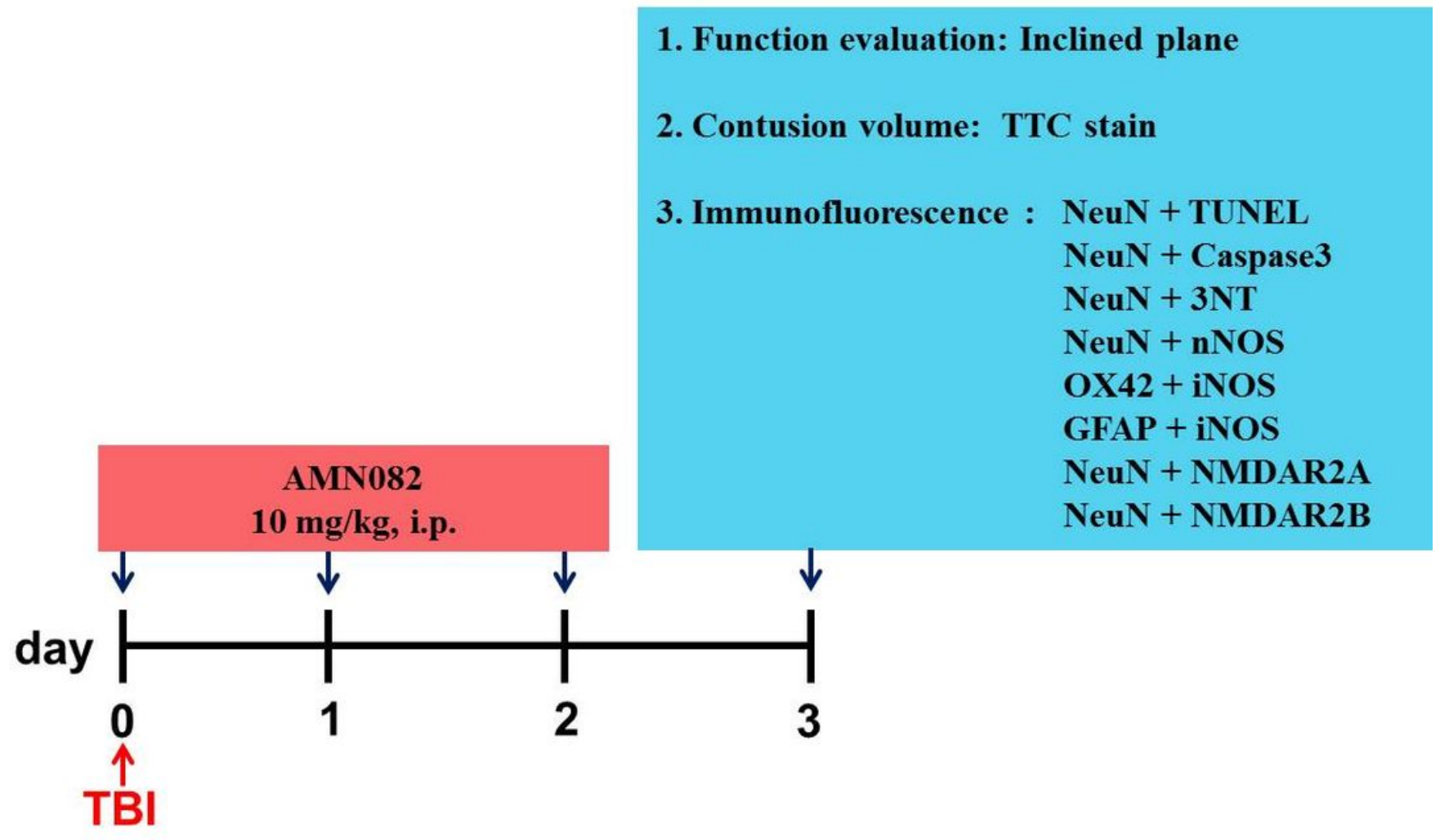

Figure 1

Overall experimental protocol. 
(A)

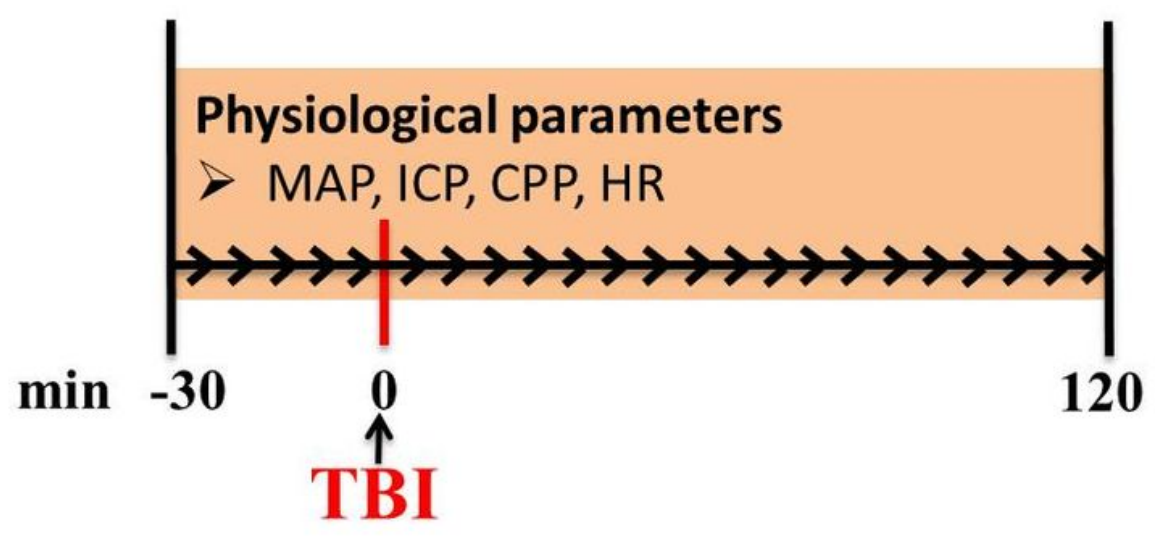

(B)

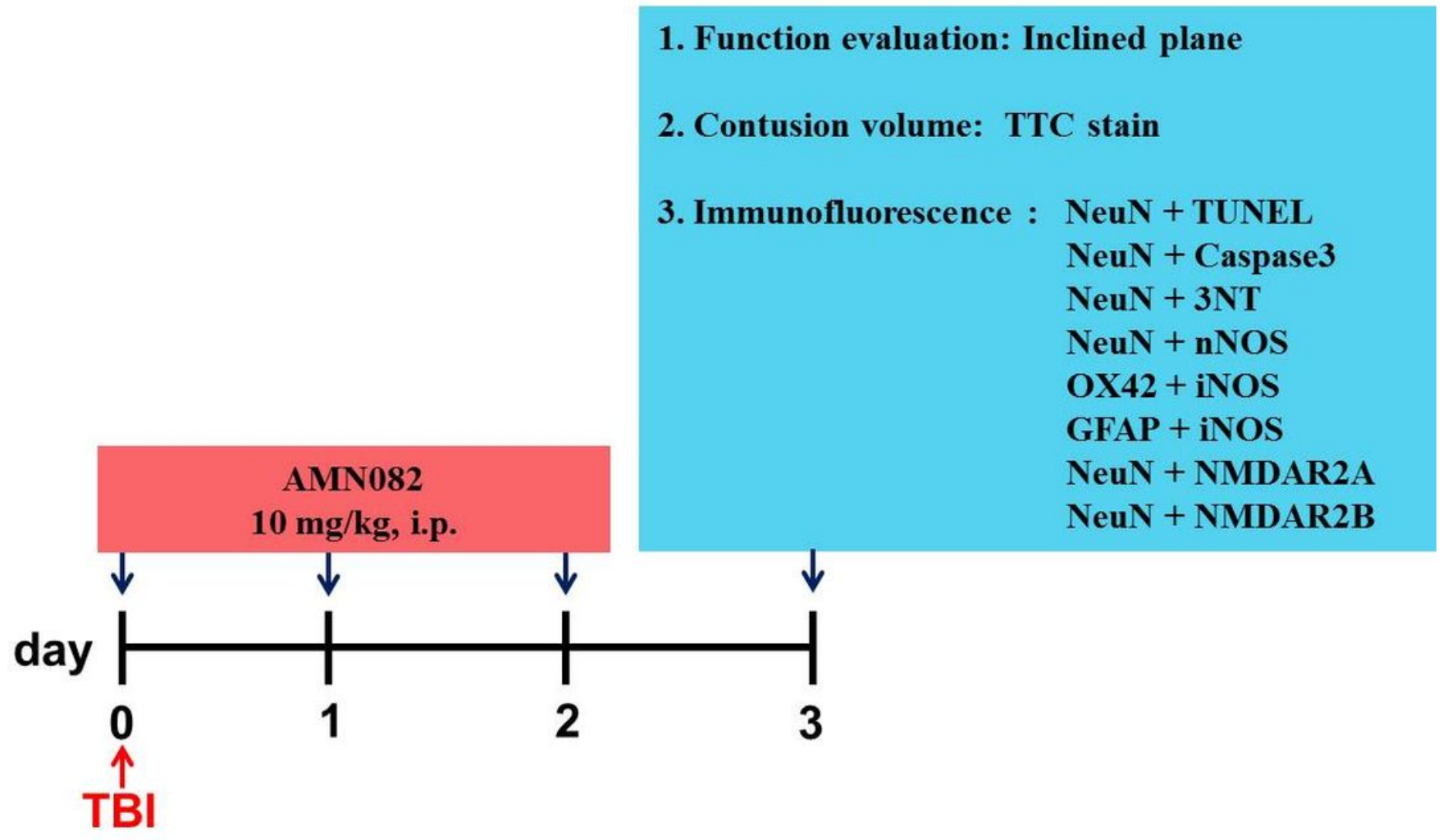

Figure 1

Overall experimental protocol. 


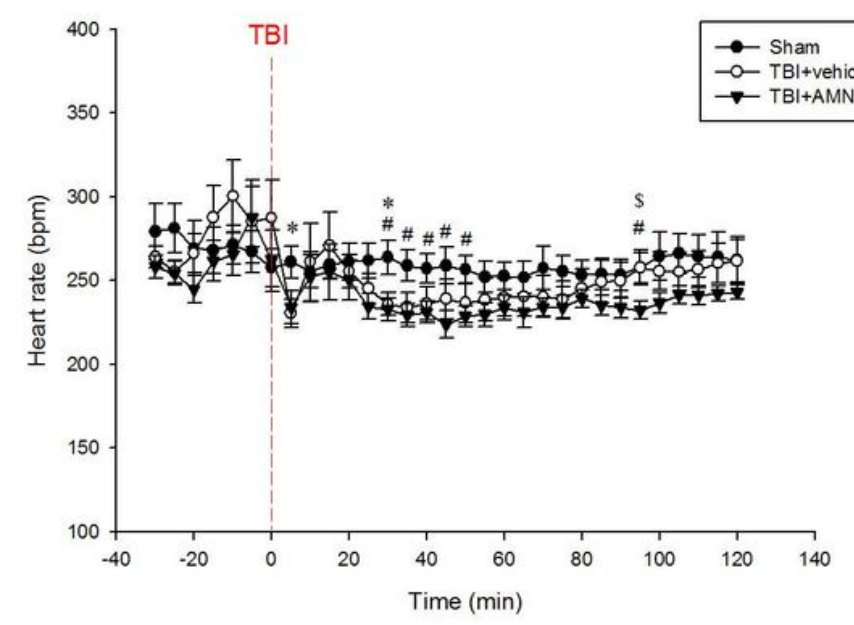

- $\mathrm{P}<0.05$, Sham compared with the TBI+vehicle

\# $\mathrm{P}<0.05$, Sham compared with the TBI+AMN082
S $\mathrm{P}<0.05, \mathrm{TBI}+$ vehicle compared with the TBI+AMN082

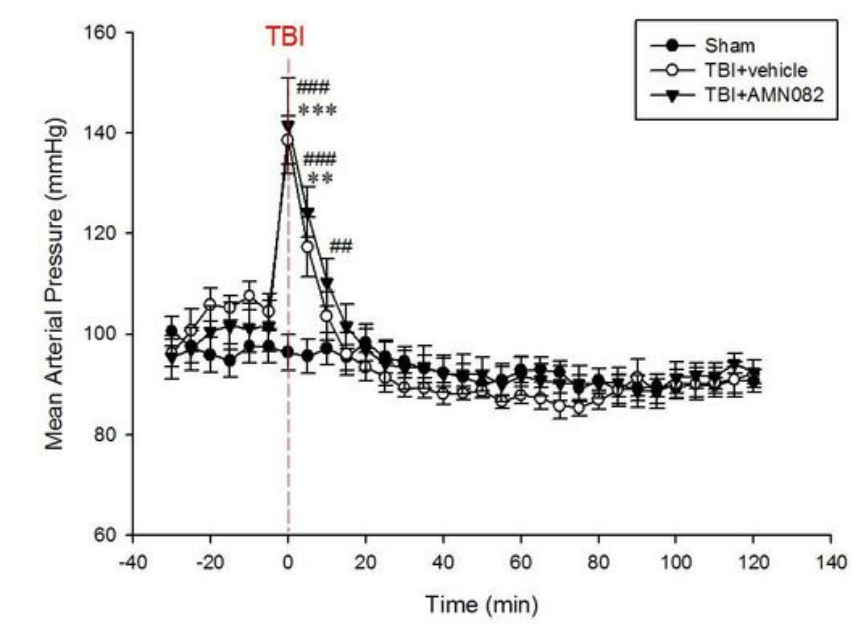

** $\mathrm{P}<0.01,{ }^{* * *} \mathrm{P}<0.001$, Sham compared with the TBI+vehicle \#\# $\mathrm{P}<0.01$, \#\#\# $\mathrm{P}<0.001$, Sham compared with the $\mathrm{TBI}+\mathrm{AMN} 082$

$2 c$

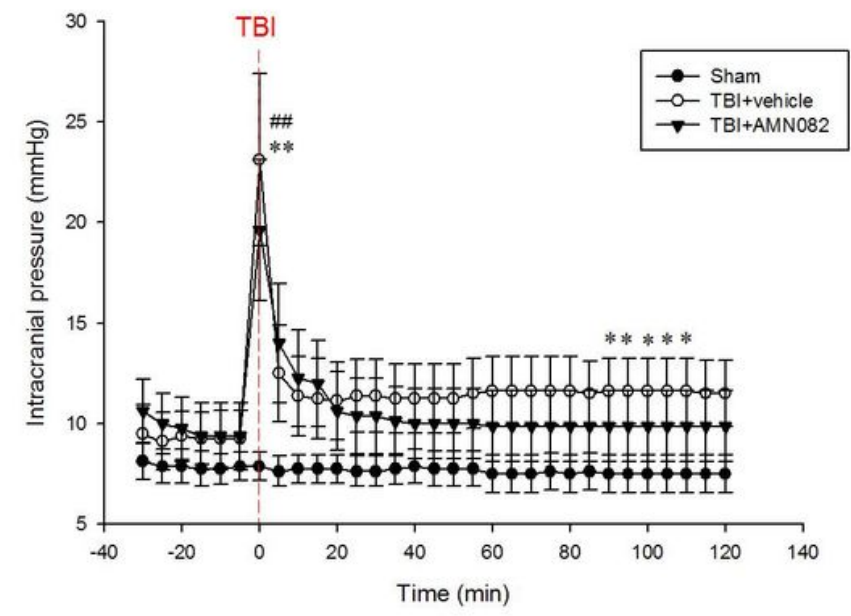

" $\mathrm{P}<0.05, * * \mathrm{P}<0.01$, Sham compared with the TBI+vehicle \#\# $\mathrm{P}<0.01$, Sham compared with the TBI+AMN082
$2 d$

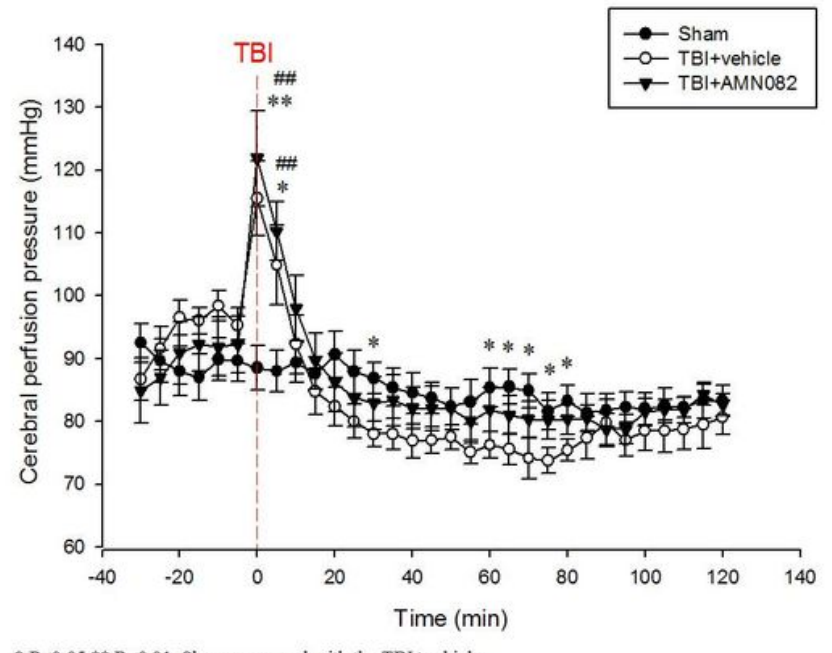

* $\mathrm{P}<0.05, * * \mathrm{P}<0.01$, Sham compared with the TBI+vehicle \# $\mathrm{P}<0.01$, Sham compared with the TBI+AMN082

\section{Figure 2}

Effects of AMN082 on TBI-induced (a) heart rate (HR), (b), mean arterial pressure (MAP),(c) intracranial pressure (ICP), and (d) cerebral perfusion pressure (CPP) during the 120 minutes after traumatic brain injury (TBI) * $\mathrm{p}<0.05$ sham compared with the TBI; $\# p<0.05$ sham compared with the TBI + AMN082; $n=$ 6 in each group 


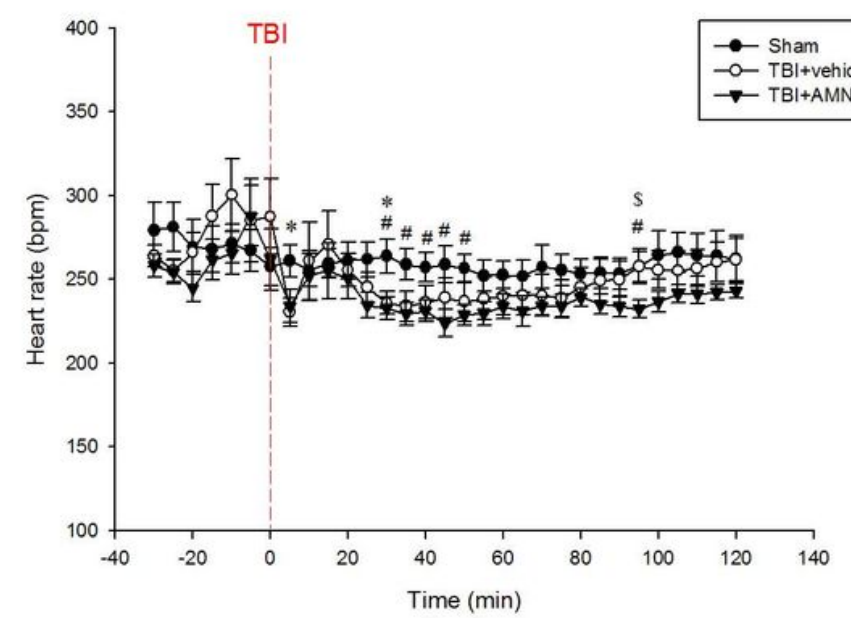

- $\mathrm{P}<0.05$, Sham compared with the TBI+vehicle

\# $\mathrm{P}<0.05$, Sham compared with the $\mathrm{TBI}+\mathrm{AMN} 082$
$\mathrm{~S}<0.05, \mathrm{TBI}+$ vehicle compared with the $\mathrm{TBI}+\mathrm{AMN} 082$

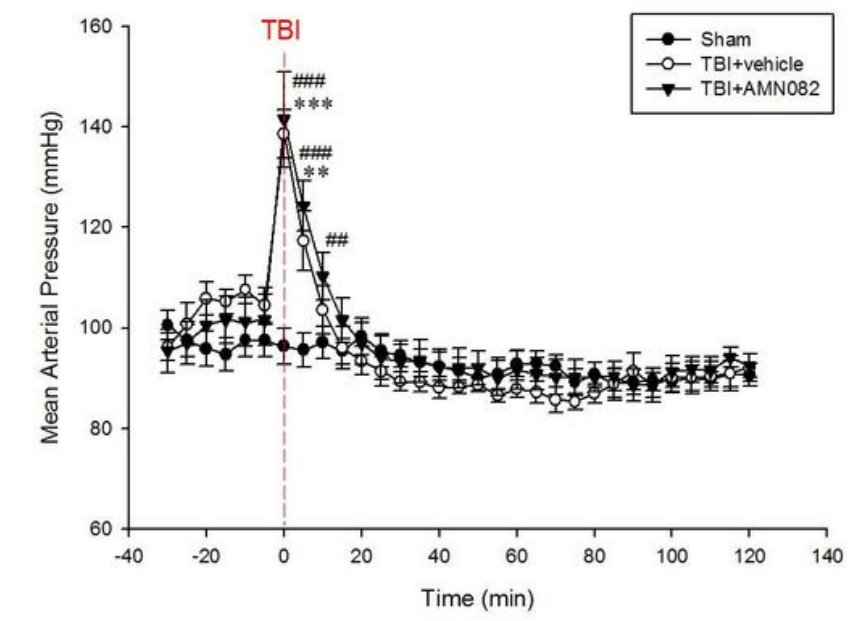

$2 c$

** $\mathrm{P}<0.01$,*** $\mathrm{P}<0.001$, Sham compared with the TBI+vehicle \#\# $\mathrm{P}<0.01$, \#\# $\mathrm{P}<0.001$, Sham compared with the $\mathrm{TBI}+\mathrm{AMN} 082$

\section{$2 d$}

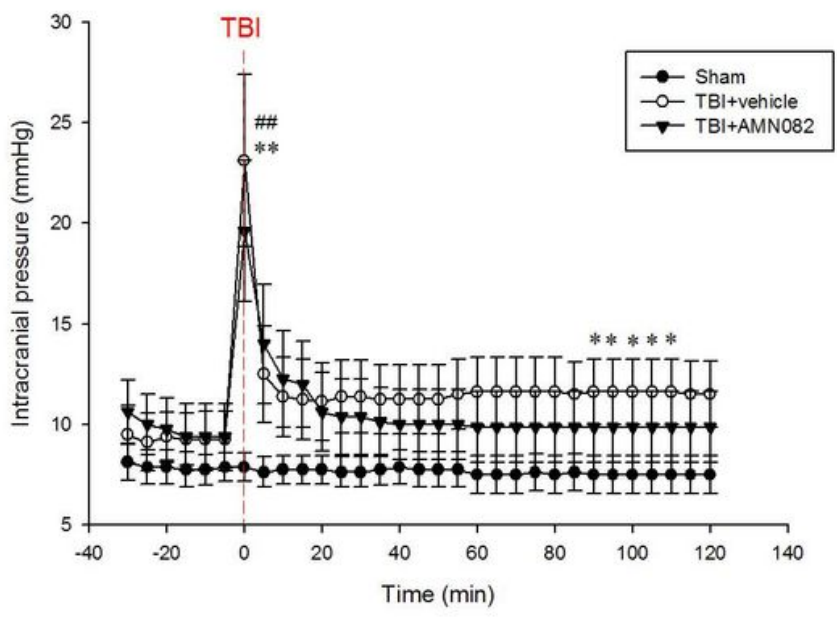

- $\mathrm{P}<0.05,{ }^{* *} \mathrm{P}<0.01$, Sham compared with the TBI+vehicle \#\# $\mathrm{P}<0.01$, Sham compared with the TBI+AMN082

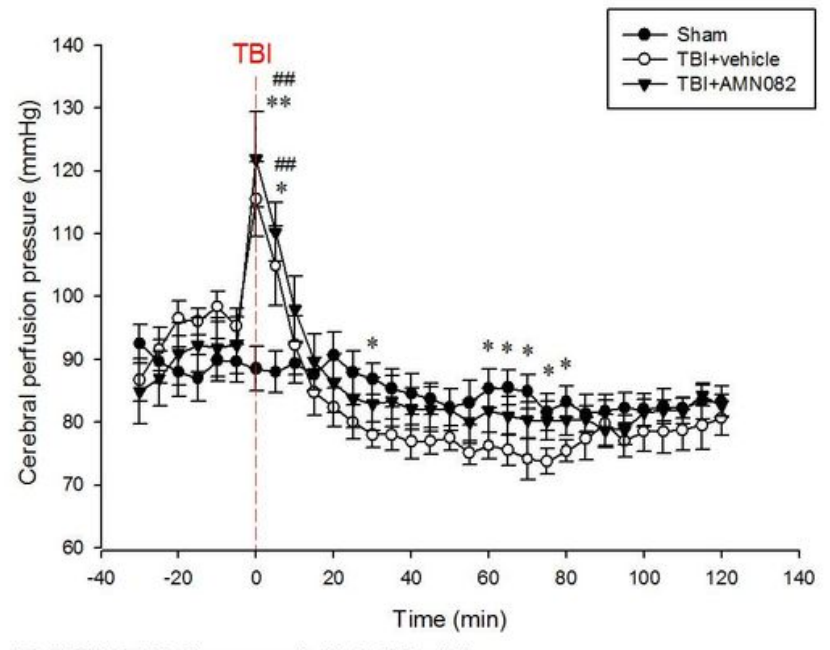

* $\mathrm{P}<0.05, * * \mathrm{P}<0.01$, Sham compared with the TBI+vehicle \# $\mathrm{P}<0.01$, Sham compared with the TBI+AMN082

Figure 2

Effects of AMN082 on TBI-induced (a) heart rate (HR), (b), mean arterial pressure (MAP),(c) intracranial pressure (ICP), and (d) cerebral perfusion pressure (CPP) during the 120 minutes after traumatic brain injury (TBI) * $\mathrm{p}<0.05$ sham compared with the TBI; $\# p<0.05$ sham compared with the TBI + AMN082; $n=$ 6 in each group 


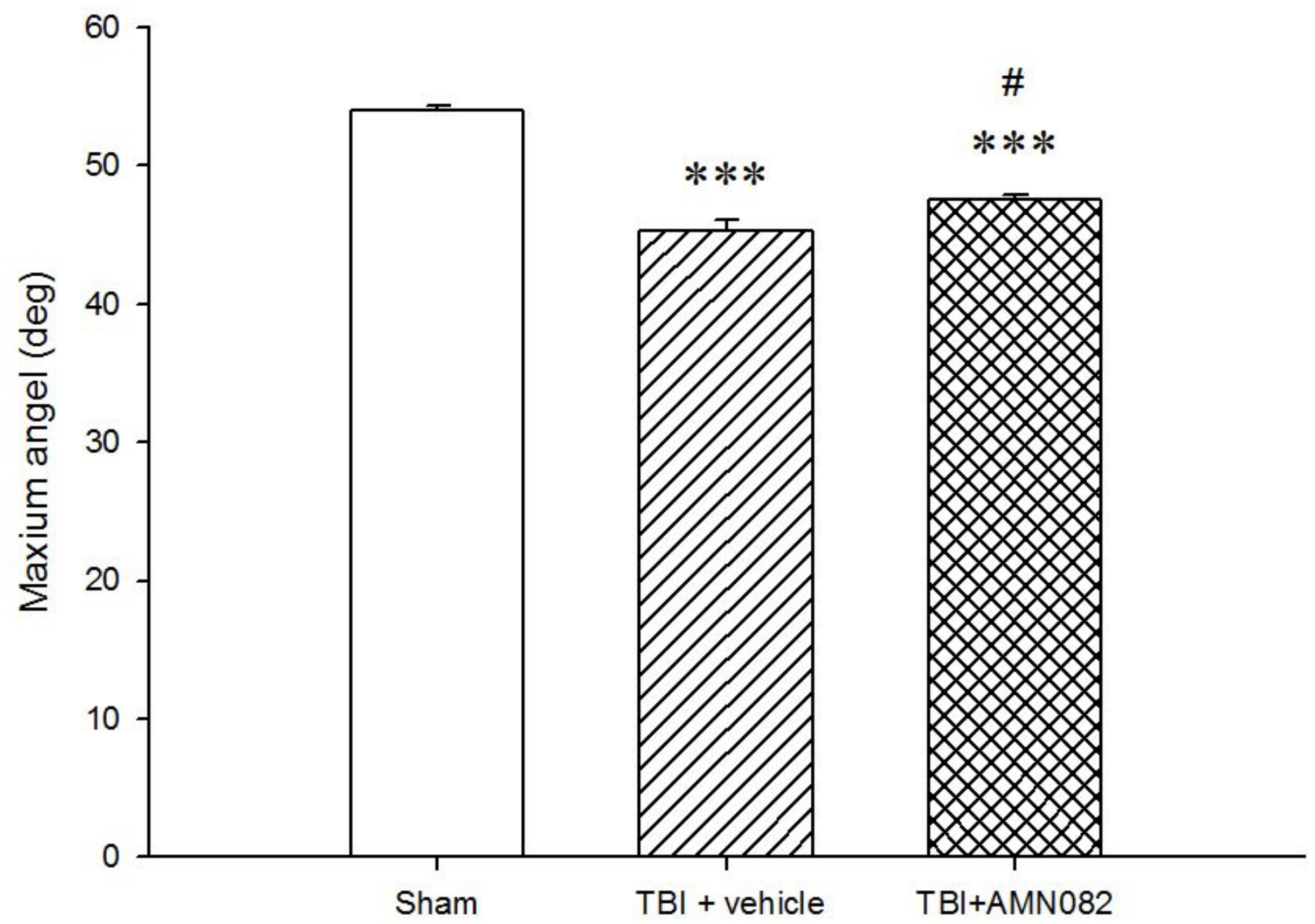

*** $\mathrm{P}<0.001$, compared with the Sham group

\# $\mathrm{P}<0.05$, compared with the $\mathrm{TBI}+$ vehicle group

Figure 3

Effects of the TBI-induced motor deficits on the 3rd day after TBI. $* \star \star p ~<0.001$, sham compared with the $\mathrm{TBl}$; \#\#\#p<0.05, sham compared with the TBI + AMN 082; $\$ p<0.005$, TBI compared with the TBI + AMN082; $n=6$ in each group. 


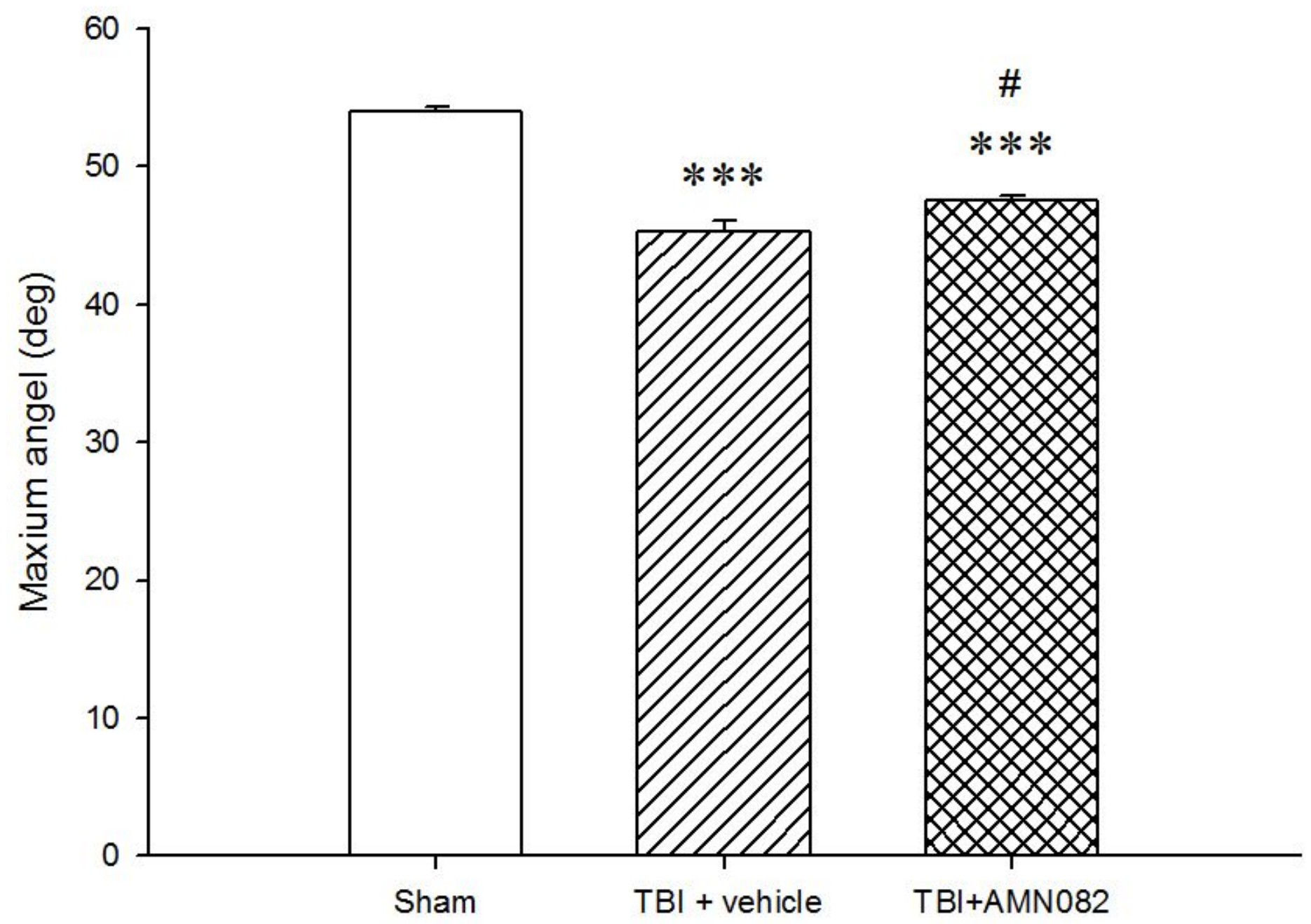

*** $\mathrm{P}<0.001$, compared with the Sham group

\# $\mathrm{P}<0.05$, compared with the $\mathrm{TBI}+$ vehicle group

Figure 3

Effects of the TBI-induced motor deficits on the 3rd day after TBI. $* \star \star p ~<0.001$, sham compared with the $T B I ; \# \# \# p<0.05$, sham compared with the TBI + AMN 082; $\$ p<0.005$, TBI compared with the TBI + AMN082; $n=6$ in each group. 
Sham
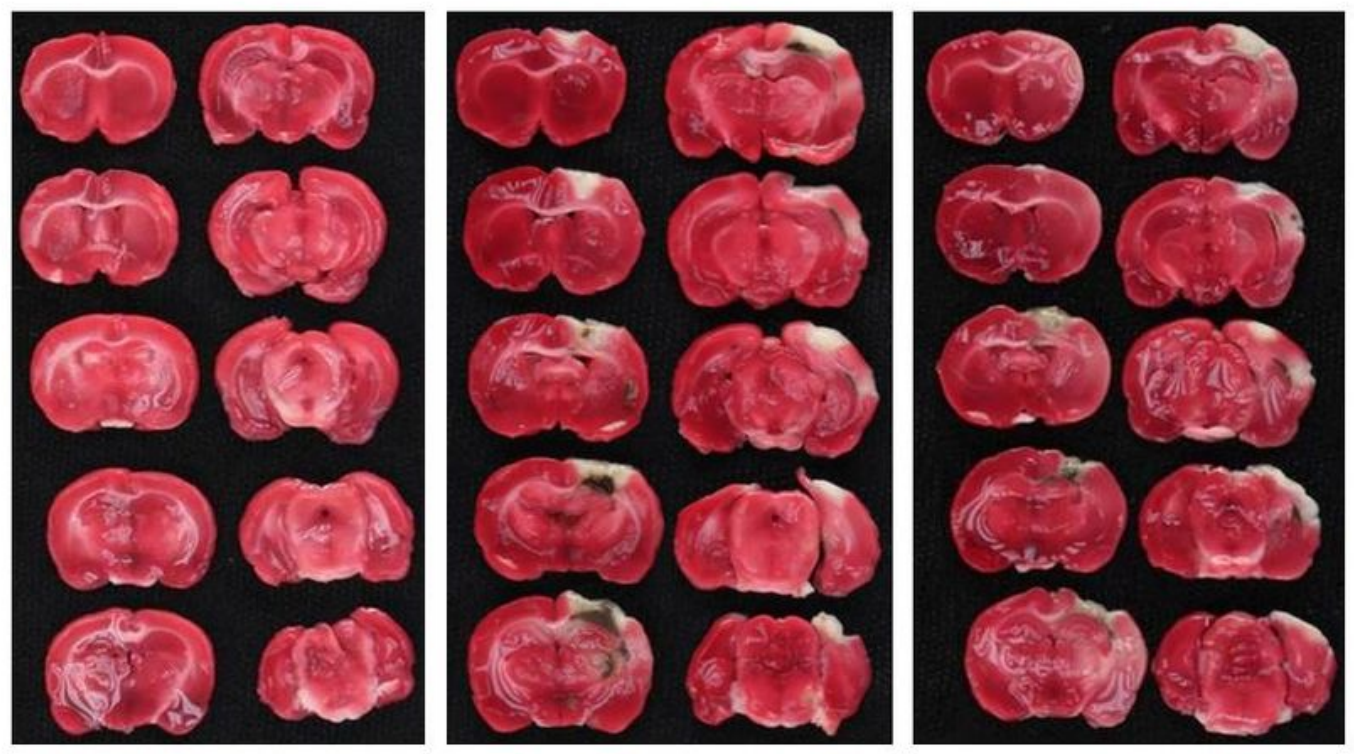

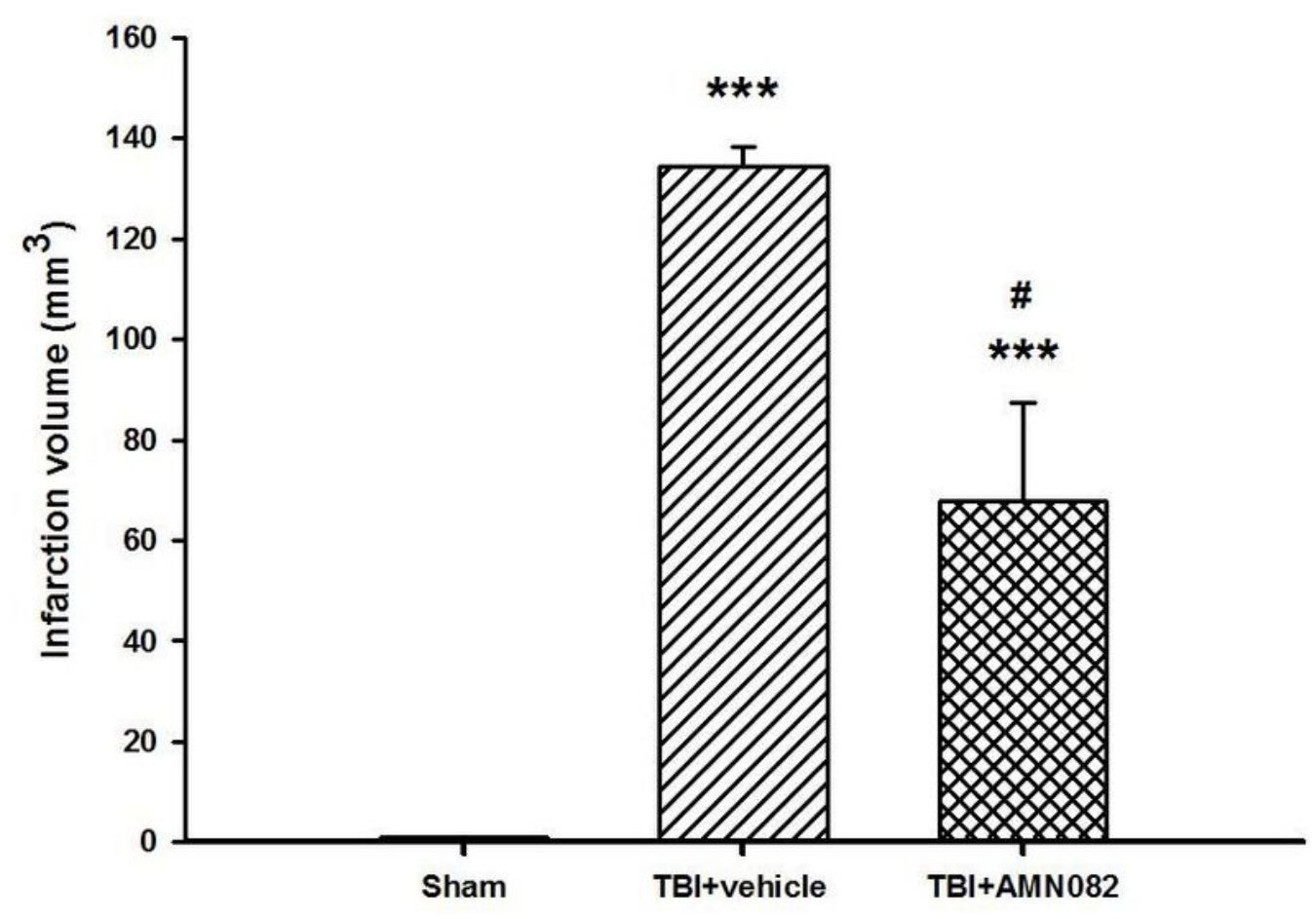

*** $\mathrm{P}<0.001$, compared with the Sham group

\# $\mathrm{P}<0.05$, compared with the $\mathrm{TBI}+$ vehicle group

\section{Figure 4}

Effects of TBI-induced infarction volumes in the ischemic cortex on the 3rd day after TBI. ${ }^{* \star} \mathrm{p}<0.001$, sham compared with the TBI; $\# p<0.05$, sham compared with the TBI + AMN082; $n=6$ in each group. 
Sham

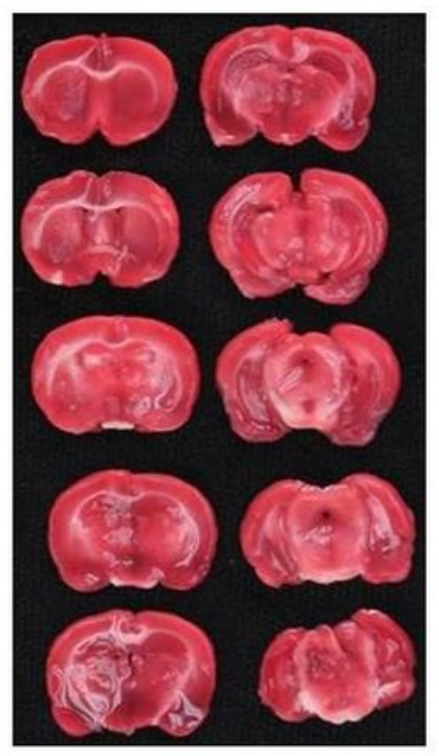

TBI+vehicle

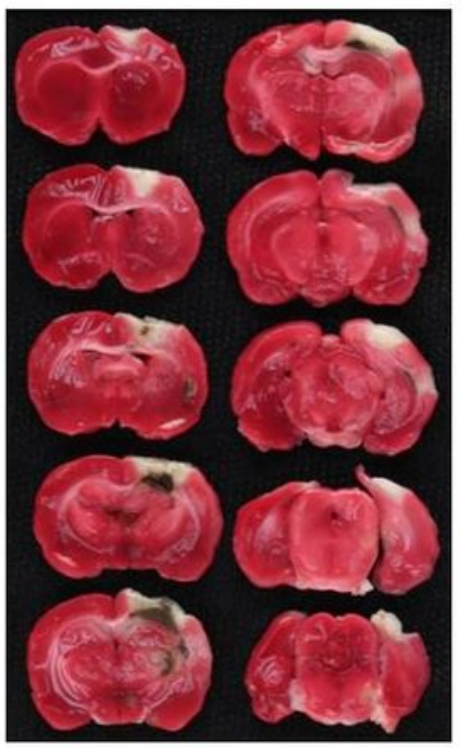

\section{TBI+AMN082}

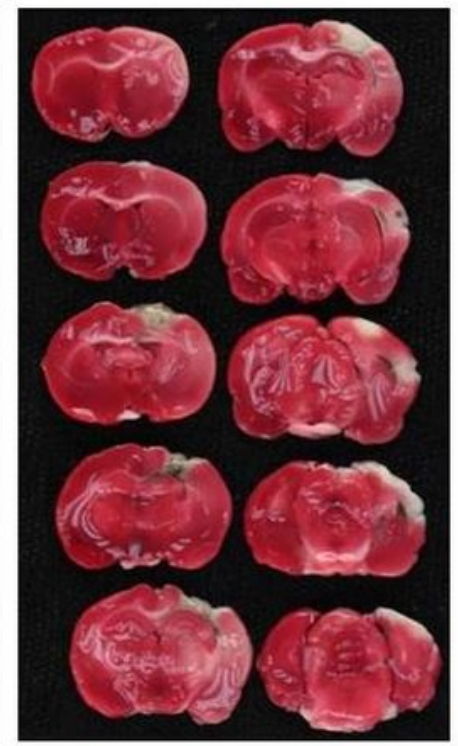

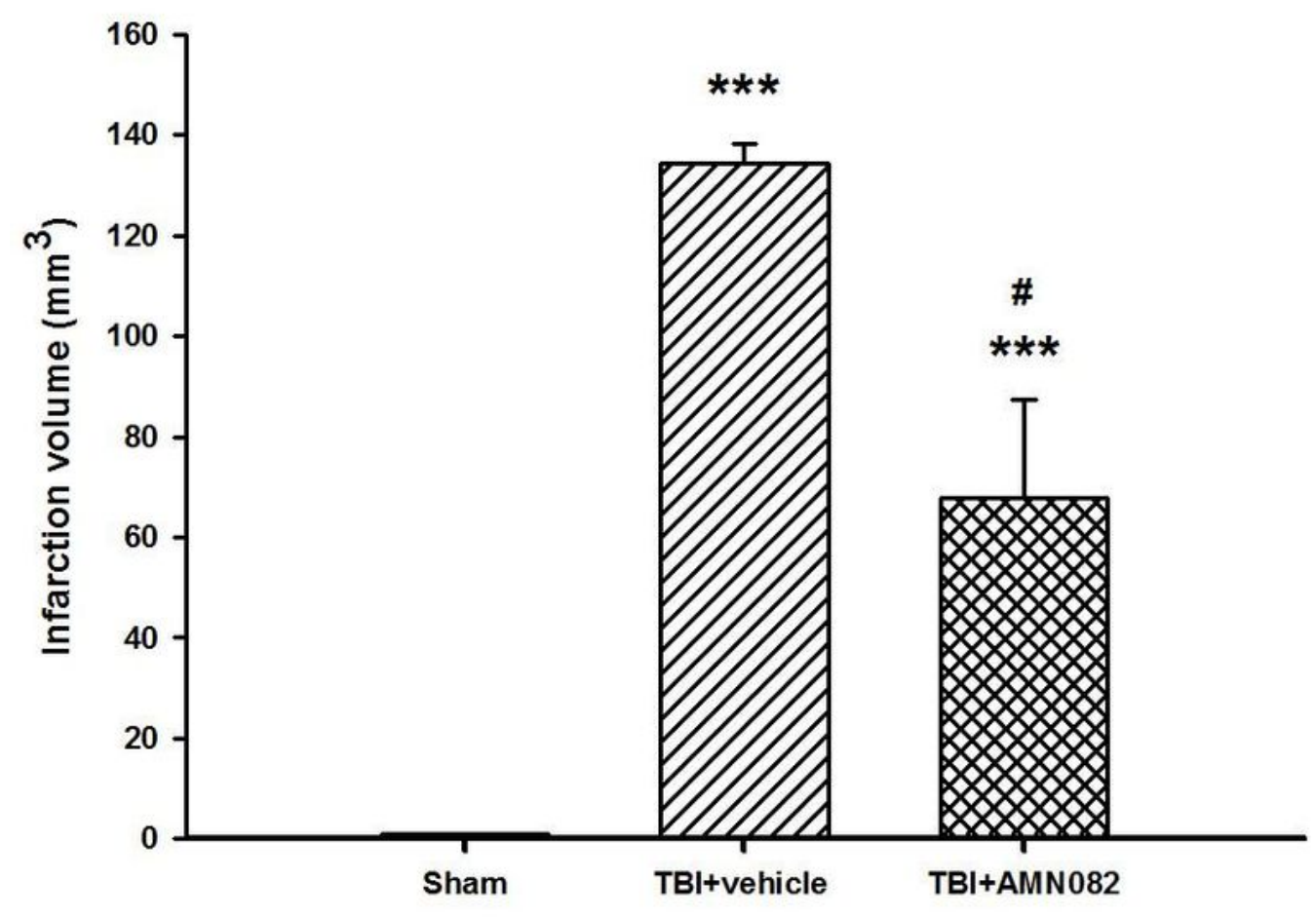

**** $\mathrm{P}<0.001$, compared with the Sham group

\# $\mathrm{P}<0.05$, compared with the $\mathrm{TBI}+$ vehicle group

Figure 4

Effects of TBI-induced infarction volumes in the ischemic cortex on the 3rd day after TBI. ${ }^{\star \star \star} \mathrm{p}<0.001$, sham compared with the TBI; $\# p<0.05$, sham compared with the TBI + AMN082; $n=6$ in each group. 

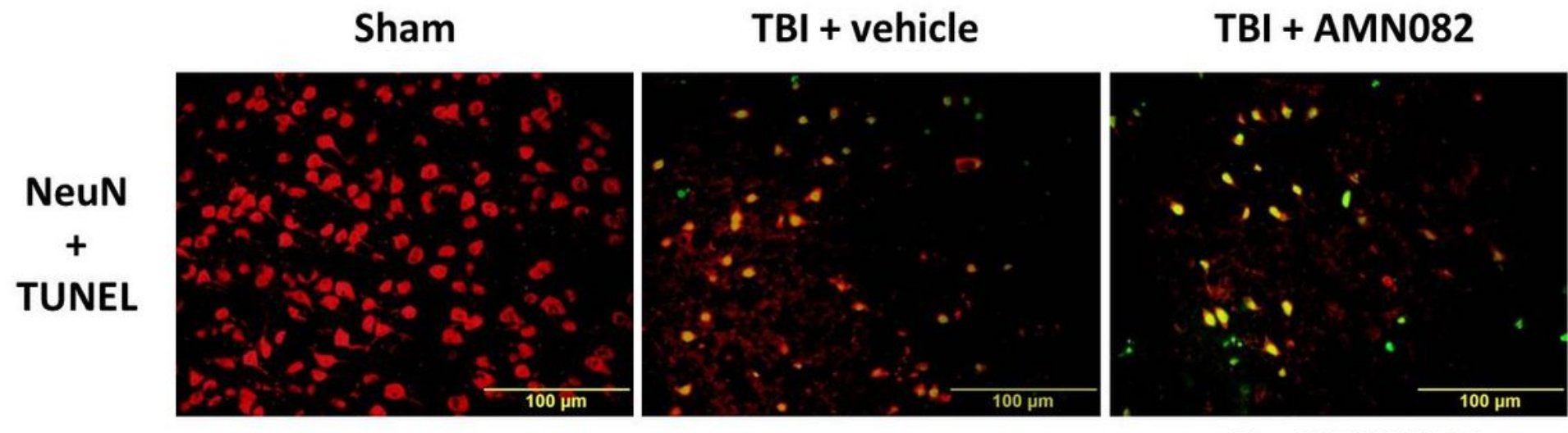

NeuN TUNEL Merge
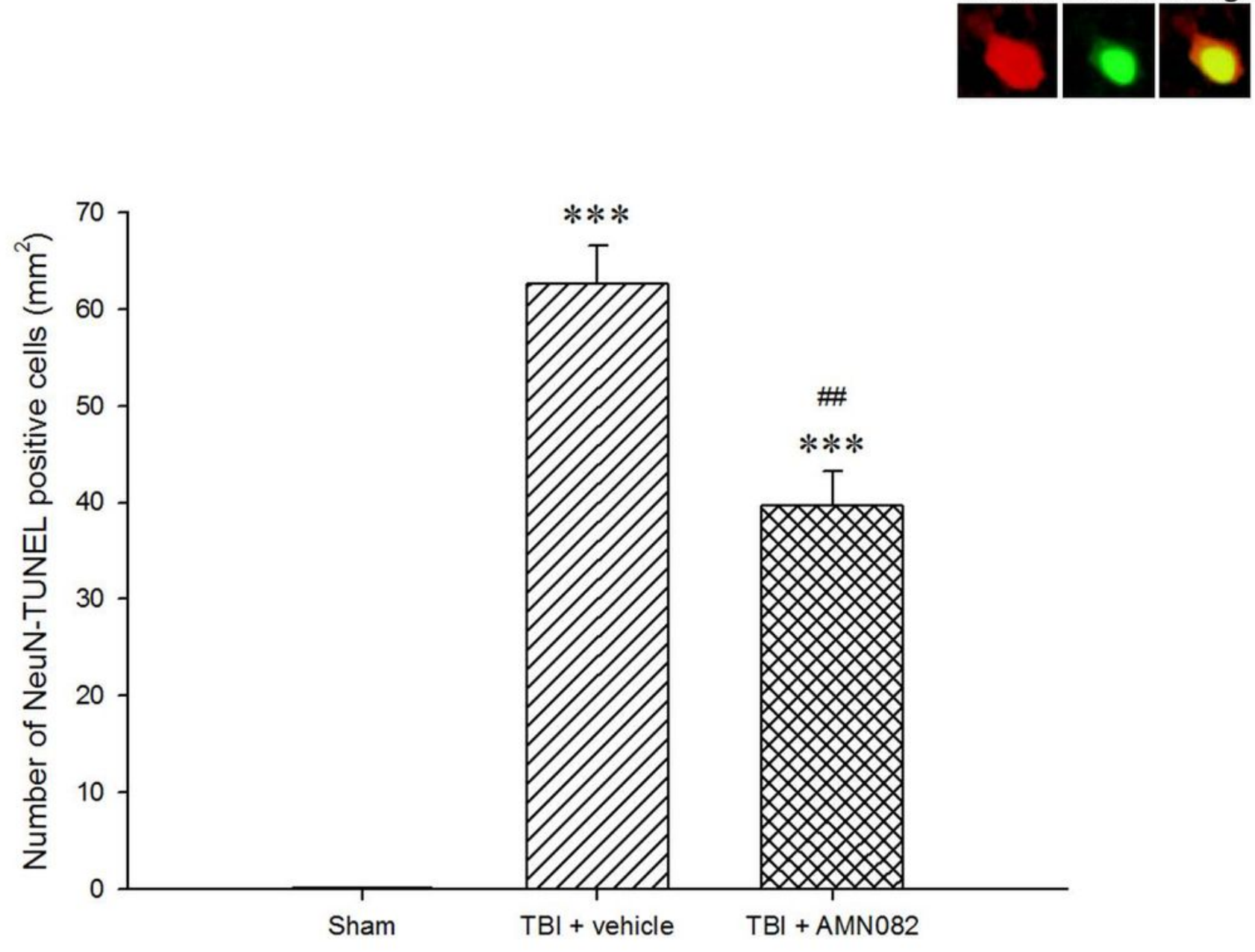

*** $\mathrm{P}<0.001$, compared with the Sham group

\# $\mathrm{P}<0.01$, compared with the $\mathrm{TB} \mid+$ vehicle group

Figure 5

Effects of TBI-induced Caspase-3 activation in neurons in the peri-contusion cortex on the 3rd day after TBI (markers Neu-N, Caspase-3), ${ }^{\star \star \star} p<0.001$, compared with sham group; $\# p<0.05$, \#\#p<0.01, compared with the TBI group; $\mathrm{n}=6$ in each group. 

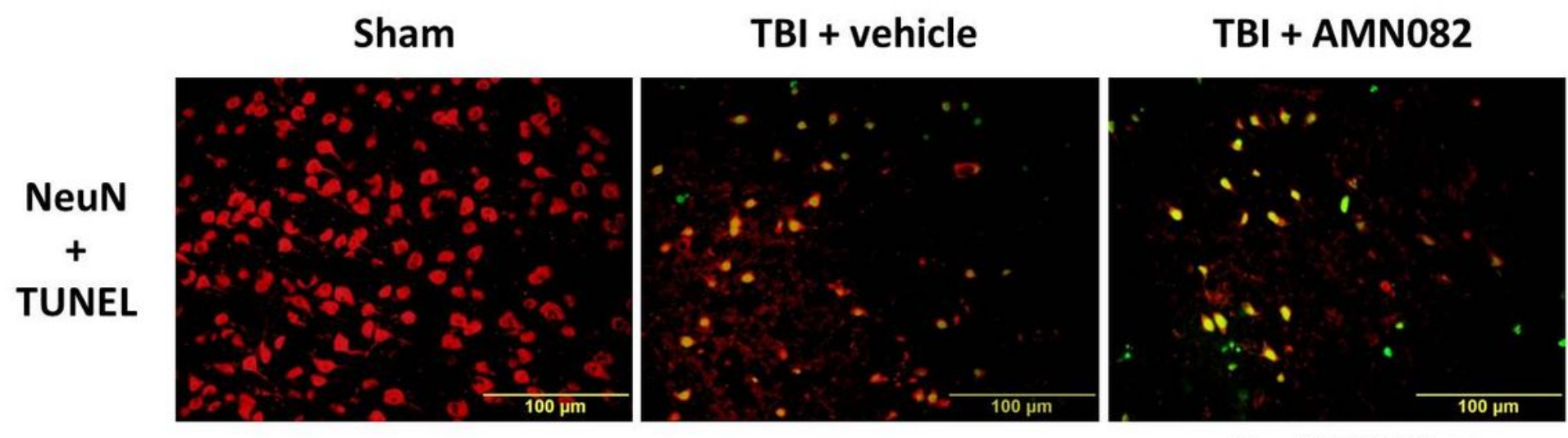

NeuN TUNEL Merge
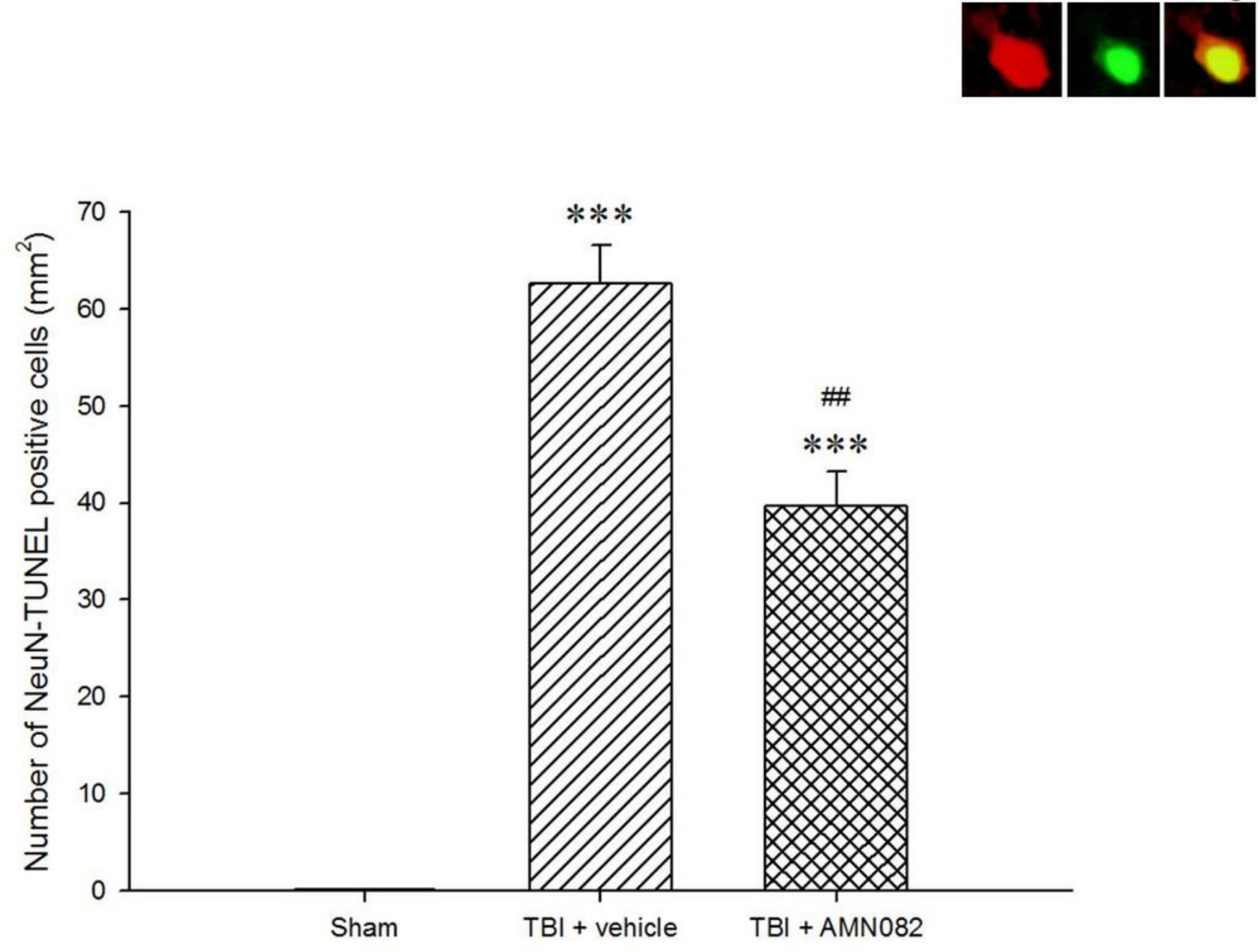

$* * * \mathrm{P}<0.001$, compared with the Sham group

\# $\mathrm{P}<0.01$, compared with the $\mathrm{TBI}+$ vehicle group

Figure 5

Effects of TBI-induced Caspase-3 activation in neurons in the peri-contusion cortex on the 3rd day after TBI (markers Neu-N, Caspase-3), ${ }^{\star \star \star} p<0.001$, compared with sham group; $\# p<0.05$, \#\#p<0.01, compared with the TBI group; $\mathrm{n}=6$ in each group. 
Sham
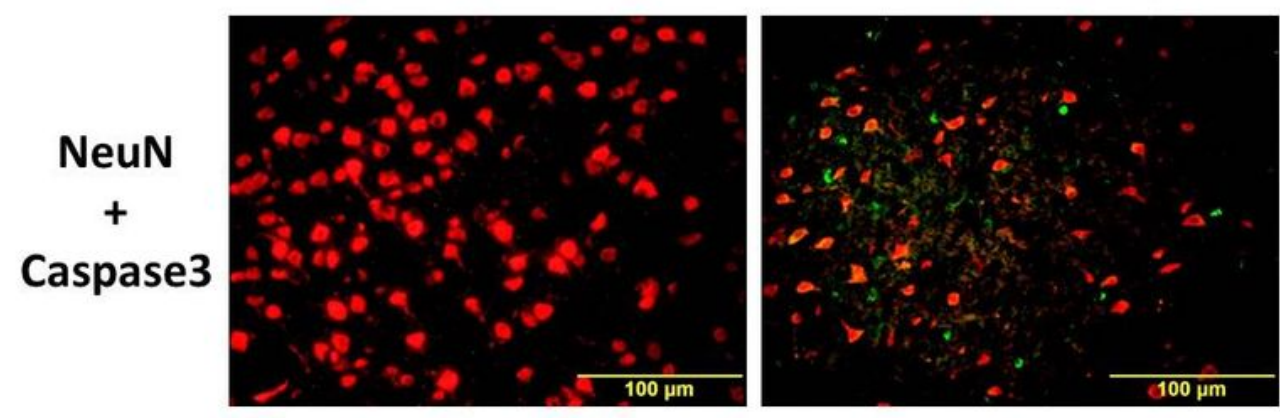

TBI + vehicle

TBI + AMN082

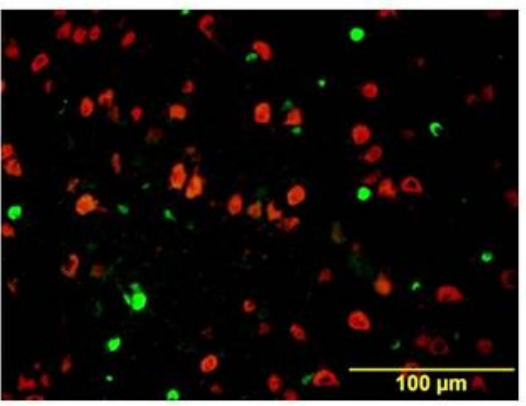

NeuN Caspase3 Merge
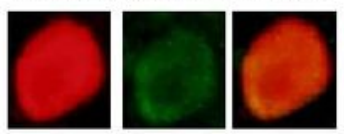

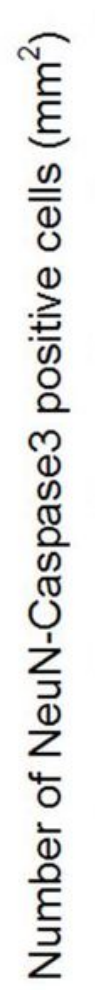

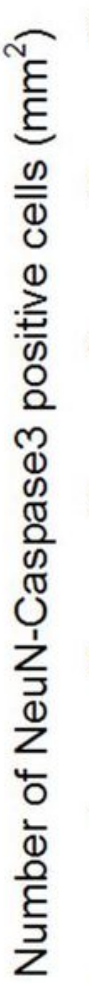

*** $\mathrm{P}<0.001$, compared with the Sham group

\# $\mathrm{P}<0.05$, compared with the $\mathrm{TB} \mid+\mathrm{vehicle}$ group
$* * *$

\#

$* * *$

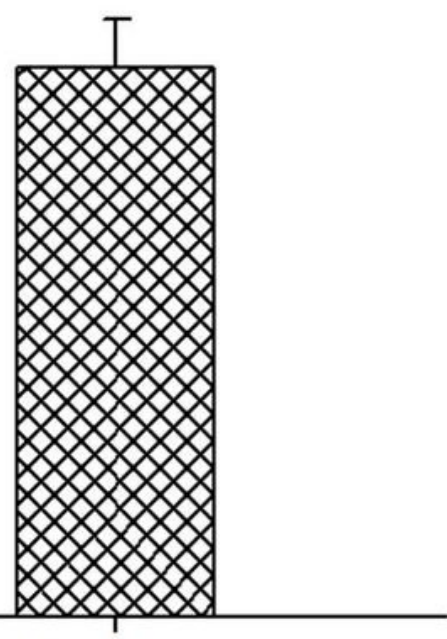

TBI + AMN082

\section{Figure 6}

Effects of TBI-induced neuronal apoptosis in the peri-contusion cortex on the 3rd day after TBI (markers

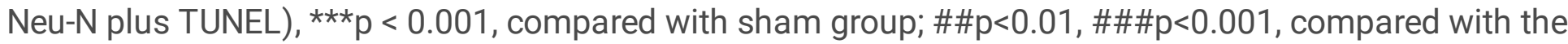
TBI group; $\mathrm{n}=6$ in each group. 
Sham
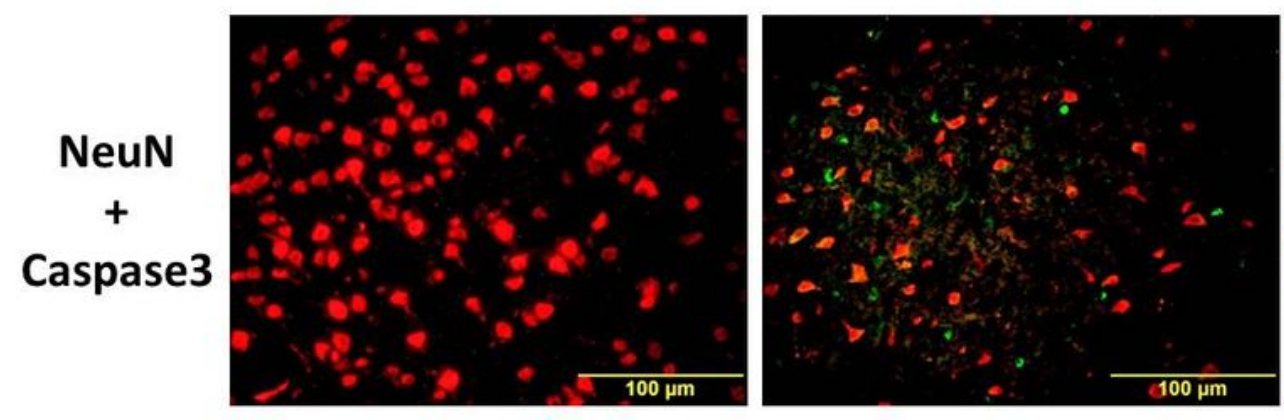

TBI + vehicle

TBI + AMN082

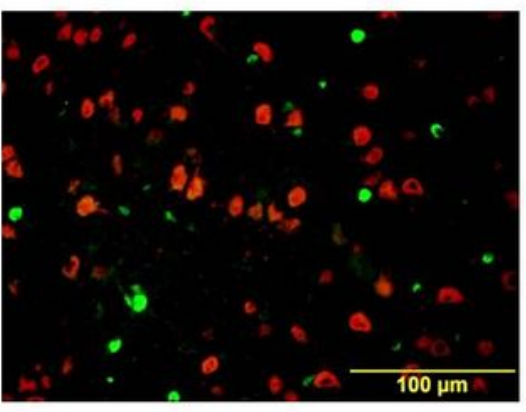

NeuN Caspase3 Merge
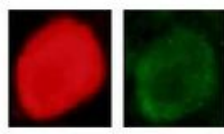

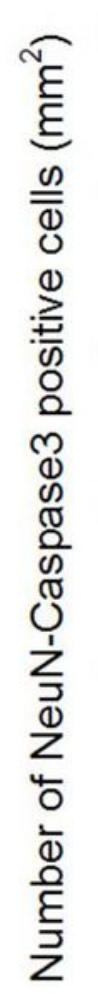

$* * *$
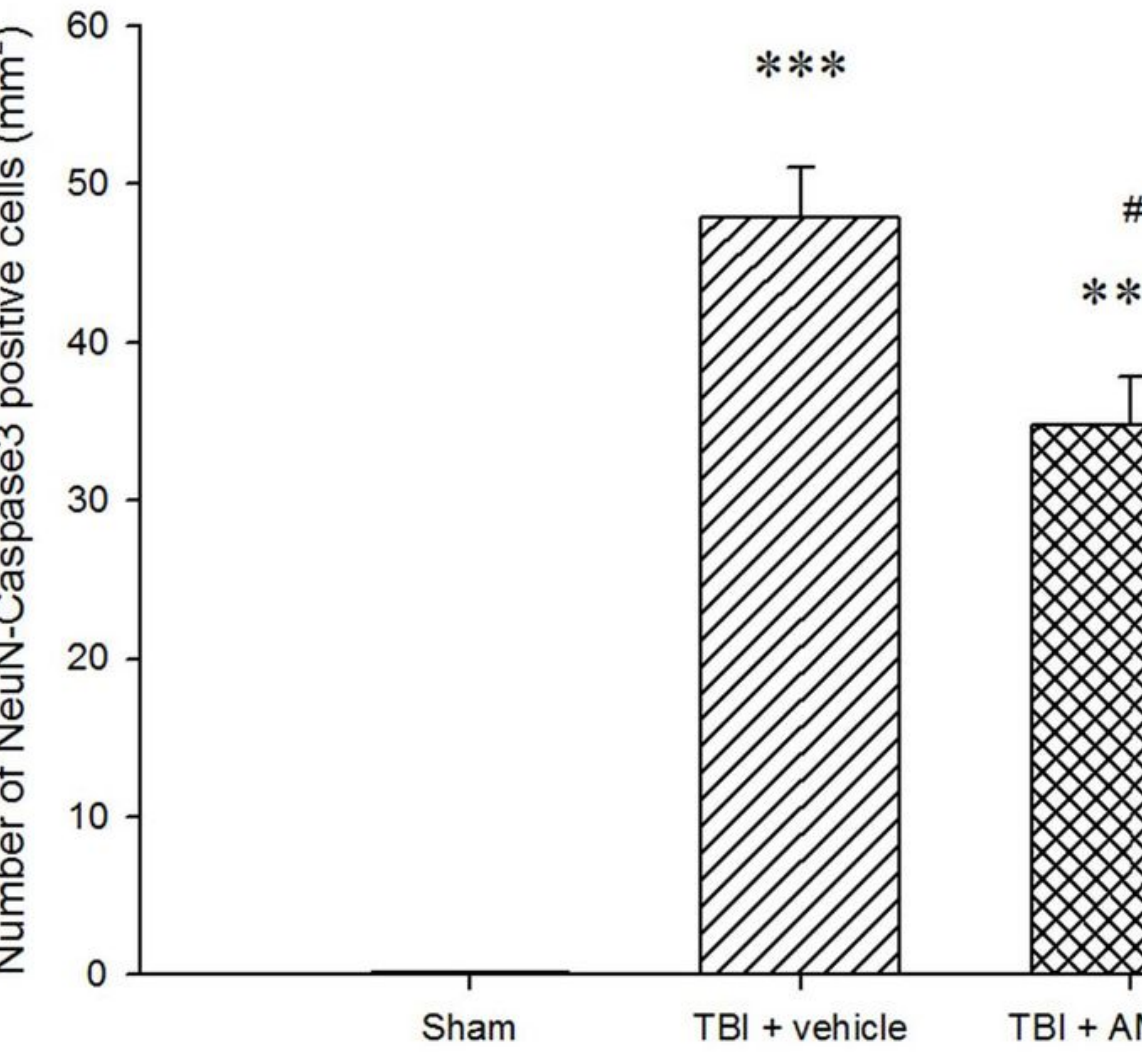

$* * *$

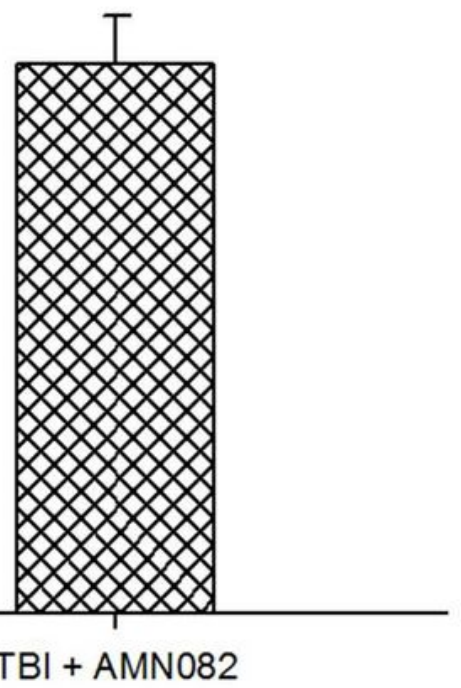

$* * * \mathrm{P}<0.001$, compared with the Sham group

\# $\mathrm{P}<0.05$, compared with the $\mathrm{TB} \mid+$ vehicle group

Figure 6

Effects of TBI-induced neuronal apoptosis in the peri-contusion cortex on the 3rd day after TBI (markers

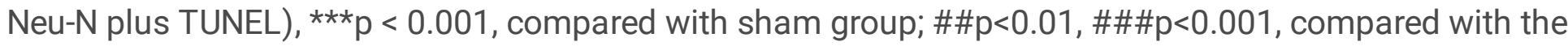
TBI group; $\mathrm{n}=6$ in each group. 